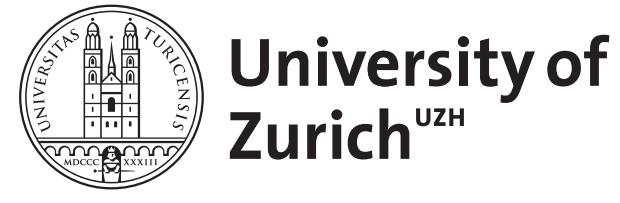
Archive

University of Zurich

University Library

Strickhofstrasse 39

CH-8057 Zurich

www.zora.uzh.ch

Year: 2003

Einleitung: Ethik zwischen Hobbes und Kant

Leist, Anton

DOI: https://doi.org/10.1515/9783110204605.1

Posted at the Zurich Open Repository and Archive, University of Zurich

ZORA URL: https://doi.org/10.5167/uzh-55334

Book Section

Published Version

Originally published at:

Leist, Anton (2003). Einleitung: Ethik zwischen Hobbes und Kant. In: Leist, Anton. Moral als Vertrag? Beiträge zum moralischen Kontraktualismus. Berlin: De Gruyter, 1-36.

DOI: https://doi.org/10.1515/9783110204605.1 


\title{
Einleitung: Ethik zwischen Hobbes und Kant*
}

\author{
Anton Leist
}

\section{Hobbes vs. Kant}

Die schärfsten Kontrahenten am Grund der Debatten in der gegenwärtigen Moralphilosophie sind Hobbes und Kant. Hobbes und Kant sind, neben Aristoteles, die wichtigsten Vorläufer unseres gegenwärtigen konflikthaften moralischen Selbstverständnisses. Bisher haben wir es kaum geschafft, die soziomoralischen und begründungslogischen Szenarien, vor die sie uns gestellt haben, auch nur vollständig auszuformulieren, geschweige zwischen ihnen zu entscheiden oder neue zu erfinden. Vieles spricht ausserdem dafür, dass es grundsätzlich andere Szenarien nicht geben kann und daß wir uns am Ende mit einer nur in den Details vertieften und vielleicht kompromißhaften Synthese beider begnügen müssen. Dieses Ende ist allerdings nicht in Sicht, denn zu ausschließlich ist einerseits der Konflikt zwischen dem hobbesianischen und dem kantischen Selbstverständnis, und zu unübersichtlich das Ausmaß der Voraussetzungen und Folgen beider. Unbegrenztes Begehren und Werthaftigkeit in sich, das sind auf eine Weise die beiden gegensätzlichen Auffassungen vom Menschen, deren Unvereinbarkeit, aber zugleich Unverzichtbarkeit unser Selbstverständnis im Alltag widersprüchlich prägen, in der Moralphilosophie, die Konsistenz anstrebt, hingegen bis heute als untergründige Antriebskräfte wirken.

Um in Konkurrenz treten zu können, müssen freilich Hintergründe geteilt werden. Was Hobbes und Kant teilen, die Frage nach der rationalen Rechtfertigung der sozialen Ordnung vor dem Individuum, ist heute allgemeines Verständnis geworden. Hobbes und Kant stoßen auf ein solches individualistisches Rechtfertigungsprinzip auf verschiedene Weise. Hobbes in Verbindung mit seinem materialistischen Wissenschaftsprogramm, motiviert auch durch die Skeptizisten der französischen Aufklärung, Kant im Rahmen der Ausarbeitung seiner kritischen Philosophie, die ihn, ausgehend von der Erkenntnistheorie, zunehmend zwingt, praktische Vernunft und Moral als die eigentlichen Grundlagen von Wissenschaft und Gesellschaft herauszustellen. Hobbes und Kant bringen sich auf durchaus ähnliche Weise in Gegensatz zur

\footnotetext{
* Für hilfreiche Hinweise und Kommentare danke ich Uwe Czaniera, Hartmut Kliemt, Thomas Schramme, Gottfried Seebaß, Holmer Steinfath sowie besonders Thomas Schmidt und Peter Stemmer.
} 
aristotelischen Philosophie sowie zur Theologie. Kant insbesondere musste sich seine eigene autonome Ethik über die Kritik der theologisch-voluntaristischen Lehre, wonach die Moral von Gott geboten ist, erst erarbeiten. ${ }^{1}$

Das tatsächliche Ausmaß der begründungslogischen Gemeinsamkeiten zwischen Hobbes und Kant wird auch deshalb leicht übersehen, weil sie im Rahmen der praktischen Philosophie unterschiedliche Interessen zu verfolgen scheinen. Hobbes gilt überwiegend als politischer, Kant als Moralphilosoph. Mit diesen Kategorien wird die Universalität beider Denkweisen jedoch verkannt. Im Unterschied zu vielen gegenwärtigen Theoretikern haben Hobbes wie Kant eine je umfassende, moralisch-politische Lösung im Sinn. Daß sie zu ganz unterschiedlichen Ergebnissen führt, steckt das Experimentierfeld der Moralphilosophie weitgehend ab. Daß und wie Hobbes und Kant eine Synthese von individuellem Handeln und Gesellschaft versuchen, ist bis heute erhellend und eben in jeweils seiner Art als Syntheseversuch vielen gegenwärtigen Ansätzen überlegen.

Die zentrale konstruktive Idee, in der sich diese Synthese bei Hobbes wirkungsträchtig präsentiert, ist der Vertrag. Obwohl Gegenstand vieler Analysen, ist diese Idee bis heute ein vieldeutiges Reservoir verschiedener Argumentationsweisen geblieben. Gegenüber Hobbes ist wiederum Kant der wichtigste ,Gegeninitiator ' des Vertragsgedankens, insofern er diese Idee in seiner politischen Philosophie konsequenter transformiert als andere ,moralische ${ }^{6}$ Kontraktualisten vor ihm, insbesondere Locke und Rousseau. Während sich Hobbes auf der einen Seite in seinem konsequenten Naturalismus von den für ihn zeitgenössischen naturrechtlichen Kontraktualisten Althusius, Grotius und Pufendorf eben dadurch abhebt, daß er jede Form religiöser Voraussetzungen in seiner Argumentation vermeidet, unterscheidet sich auf der anderen Seite Kant von den ihm nahestehenden Vertragstheoretikern Locke und Rousseau durch seine klare Trennung von Moral und Politik. Dabei nimmt er einerseits den moralischen Gehalt der Vertragsidee in ein Grundprinzip der Moral auf, den Kategorischen Imperativ, und behält andererseits, darauf gestützt, einen klar normativen Begriff des Vertrags in der Rechts-, und das heißt auch der politischen Philosophie, bei. Die Rolle dieses politischen Vertragsbegriffs wird in seiner Korrespondenz mit dem Kategorischen Imperativ sowohl ermöglicht wie gleichzeitig konstruktiv abgeschwächt.

Im Unterschied $\mathrm{zu}$ den eher kritischen als konstruktiven, der Tendenz nach eher moralischen als politischen Ansätzen des Vertragsgedankens in der Antike, ${ }^{2}$ hat die ganze moderne vertragstheoretische Tradition vor der

1 S. zu Hobbes: Watkins (1965); Goldsmith (1966); Popkin (1979) 133-148; (1982); Hampton (1986) 42-51. Zu Kant Schneewind (1998) Kap. 23.

2 S. Platon, Politeia 358e2-359b1; Epikur, bes. Kyriai Doxai; Boucher/Kelly (1994) 2-4; Gough (1936) Kap. 2; Mitsis (1988) 79-92. 
zweiten Hälfte des 20.Jahrhunderts eine dezidiert politische Ausrichtung. Der Vertragsgedanke wird von Hobbes und Locke, Rousseau und Kant dazu benutzt, um eine politische Gesellschaft oder überhaupt eine Gesellschaft, einen Souverän, eine Regierung oder die Besitzrechte zu rechtfertigen. Vertragstheorien sind vor J. Rawls überwiegend staatspolitische Theorien, in klarer Abgrenzung zu solchen der Moral. Erst Rawls versucht mit seiner Theorie der Gerechtigkeit eine Synthese, unter deutlichem Vorrang der Moral gegenüber den demokratischen Institutionen. In Anschluß an diese neuere Entwicklung will diese Einleitung - übereinstimmend mit den Artikeln dieses Bandes - einen Überblick zur aktuellen Verwendung des Vertragsgedankens in der Moralphilosophie geben.

Nötig dafür ist allerdings eine Rückbesinnung auf Hobbes und Kant. Mir scheint es ein eindrucksvoller Beleg für die außergewöhnliche Bedeutung dieser beiden Philosophen, daß gerade sie es sind, auf die sich die explizit moralphilosophische Renaissance des Vertragsgedankens zurückbezieht. Entsprechend dieses Schwerpunkts wird in dieser Einleitung sowohl von der staatspolitischen Dimension der Vertragstradition, wie von Autoren mit nur schwachem moralphilosophischen Begründungsanspruch abgesehen. Ersteres betrifft einen großen Teil des konstruktiven Inhalts der staatspolitischen Theorien von Hobbes und Locke, aber auch die neohobbesianische Verfassungsrechtfertigung von J. Buchanan (1975), letzteres erklärt, warum von Locke, Rousseau und R. Nozick (1974) nicht ausführlicher zu reden sein wird. Andererseits ist es nötig, das Verhältnis von Politik und Moral bei Hobbes und Kant im Auge zu behalten, nicht nur, weil sich anders diese Autoren gar nicht verstehen ließen, sondern auch, weil sich außerhalb von dieser Korrespondenz nicht angemessen sagen läßt, welche Funktionen die Moral und entsprechend die Moralphilosophie erfüllen können.

\section{Vertrag bei Hobbes und Kant}

Die moderne Vertragsidee in der politischen Theorie besteht darin, die Legitimität der staatlichen Ordnung anhand eines fiktiv gedachten Vertrags unter den Gesellschaftsmitgliedern zu rechtfertigen. Vor Hobbes war der Vertrag primär ein ,Herrschaftsvertrag', wurde also mit dem Herrscher geschlossen. Nach und seit Hobbes ist er primär ein ,Gesellschaftsvertrag', wird also zuerst unter den sich gleichzeitig konstituierenden Bürgern geschlossen, die ihrerseits dann einen Herrscher ermächtigen. ${ }^{3}$ Pionier ist Hobbes aber vor allem darin, daß er sich der Metapher des Vertrags nicht nur bedient, sondern sie zu einer systematischen Argumentation ausbaut, deren Bestandteile wohlbedacht sind

3 S. Euchner (1979) \$3; Tuck (1979). 
und bis heute in variierter und präzisierter Form verwendet werden. $\mathrm{Zu}$ ihnen gehören insbesondere eine naturalistische Theorie des Guten, ein hypothetisches Vertragverständnis, ein instrumenteller Vernunftbegriff, die Annahme konflikthafter Sozialbedingungen der Moral sowie deskriptiv gefaßte soziale ,Gleichheit', verstanden als gleiche Drohfähigkeit. Die naturalistische Theorie des Guten grenzt sich ab gegen das summum bonum des Aristoteles (Lev. XI, 1) und die tranquilitas des Thomas und setzt an ihre Stelle Trieb und Verlangen sowie deren Kriterien Lust und Unlust (Lev. VI, 11). Deren Ausprägung und Folgen belegt und untermauert Hobbes ebenfalls mit seiner hypothetischen Naturzustandsargumentation. Universell egoistisch sind die Menschen deshalb, weil sie sich ohne Konventionen und Gesetze in den latenten Kriegszustand hineinentwickeln würden; als gleich drohfähig und damit auf soziale Weise annähernd gleich erweisen sie sich dann, wenn die Staatsmacht jeden Einfluss verliert. Aufgrund der Mischung von grenzenlosem Begehren, Furcht und Ruhmsucht entsteht der Kriegszustand, das berühmte ,einsame, armselige, ekelhafte, tierische und kurze' Leben (Lev. XIII, 8).

Die Radikalität von Hobbes’ Vertragsargument liegt im Ausmaß sowohl seiner Sozialdiagnostik wie deren Therapie: daß die eigenständig friedensunfähigen Menschen nur mithilfe eines friedensbewahrenden absoluten Herrschers zusammenzuleben imstande sind. Obwohl diese politischen Aspekte im Vordergrund stehen, ist Hobbes' Interesse auch ein moralisches, und die modernen Vertragstheoretiker berufen sich nicht zu Unrecht auf ihn auch als Moralphilosophen. Sicher will Hobbes eine gesellschaftliche und staatliche Ordnung begründen, aber im Unterschied zu anderen vor und nach ihm bezieht er die Moral in die Grundantriebe des menschlichen Handelns nicht bereits ein, sondern hält sie für ebenso begründungsbedürftig wie das Recht und die staatliche Macht. Angesichts des sozialen Unmuts bei philosophischen Tabubrüchen noch am Ende des 20.Jahrhunderts läßt sich die Kühnheit eines Standpunkts im 17.Jahrhundert erahnen, wonach die Moral, also die Gebote des christlichen Dekalogs, trotz bestehender Gläubigkeit keineswegs sicher sind und einer Rechtfertigung unter Aspekten des primitiven Lebensinteresses bedürfen. Und nicht nur das: selbst so geklärt sind die moralischen Gebote nur dann sozial wirksam, wenn sie durch das über ihnen hängende Schwert geschützt werden.

In nuce entwirft Hobbes jedoch die erste vertragstheoretische Rekonstruktion der Gebote des Dekalogs. Ihnen voran stehen die beiden ersten ,natürlichen Gesetze , in denen sich die typische Logik einer selbstinteressierten Argumentation ausdrückt: weil der Naturzustand in niemandes Interesse ist, gebietet die Vernunft, daß sich jeder, wenn möglich, um Frieden bemühen soll sowie daß jeder seine natürliche Freiheit (sein natürliches ,Recht auf alles $^{\mathrm{c}}$ ) soweit einschränken soll, wie es mit der gleichen Freiheit der anderen vereinbar ist (Lev. XIV, 4-5). Alle weiteren natürlichen Gesetze und die in ihnen ausgedrückten traditionellen Tugenden werden von Hobbes auf der 
Grundlage dieser Friedensgebote als „Mittel zu einem friedlichen, geselligen und bequemen Leben“ (Lev. XV, 40) gepriesen und so als Bestandstücke eines konsequent durchrationalisierten Lebensinteresses geschildert.

Hobbes' Überzeugung, daß die moralischen Gesetze ohne eine Delegation der Macht an den Souverän nicht eingehalten würden, bringt allerdings im Anschluß an diesen ersten Schritt einer Rechtfertigung eine nachfolgende Entkräftung der Moral mit sich, eben die Auflösung der Moral in positives Recht. Hobbes hat im Laufe seiner Schriften seine Terminologie leicht verändert, so daß Unklarheit darüber entstehen konnte, ob die Übertragung der natürlichen Rechte an den Souverän auch noch im Leviathan, der spätesten Schrift, als die kompromißlose Aufgabe dieser Rechte gemeint ist und die Vergleiche der Untertanen mit Sklaven wörtlich zu nehmen sind (Lev. XXI, 8). ${ }^{4}$ Aber es scheint naheliegend, daß die von Hobbes befürchteten Interpretationskonflikte bei einer nur partiellen Übertragung der Rechte an den Souverän, oder bei der autoritativen Rückbindung von dessen Herrschaft an die Bürger, soweit anhalten müssten, daß der Naturzustand nicht wirklich verlassen werden könnte. Im Vertrag müssen sich deshalb die Bürger gegenüber dem Souverän kompromißlos unterwerfen. Der Souverän ist frei, positive Rechte zu erzeugen, denen sie sich ihrerseits nur im Fall ihrer Lebensbedrohung widersetzen können (Lev. XVIII). Das positive, bürgerliche Recht, dem der Souverän selbst nicht unterliegt, ersetzt für alle Untertanen die Moral. Sich als Privatmann herauszunehmen, aufgrund des eigenen Gewissens Handlungen moralisch zu beurteilen, muß dementsprechend bereits als staatsgefährdend gelten (Lev. XXIX, 6-7).

Verschiedene Faktoren spielen in Hobbes' Argument zusammen und könnten kritisch revidiert oder verworfen werden. Zentral sind jedoch auf der einen Seite seine deskriptiven Annahmen über das Verhalten und die psychologischen Grundmotive von durchschnittlichen Menschen. Herausragend sind Hobbes' Individualismus, also die Annahme, daß sich die wichtigsten zur Gesellschaft befähigenden Eigenschaften nicht selbst der Gesellschaft verdanken, der Vorrang des Selbstinteresses oder des Egoismus, und die unter extremen Konflikten dominanten Eigenschaften von Furcht und Ruhmsucht. ${ }^{5}$ Auf der anderen Seite sind diese deskriptiven Annahmen eingebettet in ein insgesamt normativ gemeintes Argument, das seinerseits Ausdruck von Hobbes' Vernunftmethode ist. Deskriptive Annahmen und normative Absicht sind miteinander verknüpft. Das Vertragsargument ist explizit hypothetisch gemeint,

4 S. hierzu Hampton (1986) Kap. 5.

5 Zu diesen Faktoren detaillierter Hampton (1986) Kap. 1. Inwieweit Hobbes eine egoistische Psychologie vertrat, ist umstritten: s. die Zusammenfassung bei Hampton (1986) 19-24 und Kavka (1986) 44-51. 
Naturzustand und Vertrag sind nicht als historische Ereignisse gedacht (Lev. XIII, 11). Dennoch dient die hypothetische Überlegung auch dazu, einen real drohenden Naturzustand zu veranschaulichen, sie steht also nicht im Gegensatz zu den deskriptiven Behauptungen. Da auf diese Weise selbst noch die den Menschen zugeschriebenen Grundeigenschaften hypothetisch-real sind, scheint Hobbes' Psychologie, so bedeutsam sie für die Gesamtargumentation auch ist, schwer überprüfbar.

Eine Möglichkeit, auf dieses Problem zu reagieren, besteht darin, die hypothetische Argumentation soweit zu präzisieren, daß sie mit vergleichsweise schwachen und allgemeingültigen psychologischen Prämissen zu ähnlichen Ergebnissen gelangt, wie sie Hobbes vorgeschwebt haben. Neuere Autoren wie G. Kavka und D. Gauthier gehen diesen Weg, indem sie den ,durchschnittlichen', der Gesellschaft im ganzen gegenüber akzeptablen und vor allem auf dem Markt allseits akzeptierten Egoismus verteidigen oder sogar als sozial vorteilhaft schildern. ${ }^{6}$ Eine Stärke dieses Neohobbesianismus scheint auch darin zu liegen, daß ein bestimmtes, als real verbreitet angenommenes $\mathrm{Ma}$ an Egoismus akzeptiert werden kann, um darauf eine moralisch-rechtliche Ordnung zu bauen, während, zumindest aus der Sicht dieser Position, alternative Theorien ein größeres Ausmaß an Altruismus unterstellen müssten. Das ,Risiko' der deskriptiven Annahmen scheint dann bei den Kritikern zu liegen. Eine ganz andere Sichtweise ergibt sich jedoch dann, wenn man den Vernunftbegriff von Hobbes ablehnt und sich dementsprechend weigert, den ,Realismus' philosophischer Argumente einzig anhand von psychologischen Prämissen, also des tatsächlichen Ausmaßes von Egoismus oder Altruismus zu messen. Diese alternative Sichtweise ist diejenige von Kant und des ihm heute folgenden Neokantianismus.

Kants deskriptive Äusserungen zu den psychologischen Eigenschaften von durchschnittlichen Menschen erscheinen in einer Hinsicht nicht so völlig anders als diejenigen von Hobbes. Neigungen und Begehren sind in Lust und Unlust fundiert und als solche nicht stark beeinflußbar. Glückseligkeit kann nur erfahren, nicht eigentlich erkannt werden (GMS, 418-419). Dabei kann die Glückseligkeit nie erreicht werden, die menschliche Natur ,ist nicht von der Art, irgendwo im Besitze und Genusse aufzuhören und befriedigt zu werden“ (KU, $\mathbb{S} 83)$. Ehrsucht, Herrschsucht und Habsucht gehören zu den prägenden menschlichen Eigenschaften. Ehre, Gewalt und Geld werden als das Vermögen analysiert, über andere Macht auszuüben (Anth, $\mathbb{S}$ 79-82). Gewalt und Zwang sind nötig, um die bösartigen Neigungen unter Kontrolle zu bringen. Neben der ,Tugendlehre', der inhaltlichen Ausgestaltung der Kant-

6 Kavka (1986) 64-80; Gauthier (1986) Kap. 4. 
schen Ethik, ist natürlich die ,Rechtslehre', die auf, äusserem' Zwang beruht und nicht, wie jene, auf ,innerer' Motivation, nicht weniger wichtig.

Trotz dieser vergleichsweise hobbesianischen Schilderung der menschlichen Eigenschaften entwickelt Kant ein völlig anderes Moral-, Staats-, Politik- und Geschichtsverständnis als Hobbes, indem er radikaler als je zuvor die Vernunftfähigkeiten von den Leidenschaften abkoppelt und ihnen eine Eigenständigkeit einräumt, die zu dramatisch veränderten Szenarien in Moral und Politik führt. Grundlage ist ein Vernunft- und Freiheitsbegriff, der den Leidenschaften nicht mehr zu- oder sogar untergeordnet ist, sondern der, psychologisch irreduzibel, auch eine eigenständige Dynamik der Vergesellschaftung zu entwickeln vermag, entgegen aller naturbedingten Widrigkeiten. Diese Eigenständigkeit bewirkt, daß die Moral nicht in Politik aufgelöst werden muß, sondern unabhängig von ihr bestehen bleiben kann und sie vielmehr ebensowohl fundiert wie kontrolliert.

Obwohl Kant rein begrifflich am Gedanken eines politischen Vertrags festhält, spielt für ihn ein Vertrag innerhalb eines normativen Arguments keine konstruktive Rolle mehr. Da die Moral bereits unabhängig von einem Gesellschaftsvertrag in der praktischen Vernunft fundiert ist, wird der Vertrag als eine moralische Metapher, und ohne Begründungsabsicht, verwendet. Ein Vertrag muß nicht mehr, auch nicht mehr idealerweise, vollzogen werden, sondern ist im ,moralischen Gesetz' bereits vorweggenommen, das auch der Politik gegenüber zur eigentlichen Argumentationsbasis wird. Mit der Rede vom Vertrag zollt Kant nur noch der Tradition einen terminologischen Tribut, wobei aber normativ immer das moralische Gesetz gemeint ist.

„Hier ist nun ein ursprünglicher Kontrakt, auf den allein eine bürgerliche, mithin
durchgängig rechtliche Verfassung unter Menschen gegründet und ein gemei-
nes Wesen errichtet werden kann. - Allein dieser Vertrag ... als Koalition jedes
besonderen und Privatwillens in einem Volk zu einem gemeinschaftlichen und
öffentlichen Willen ..., ist keineswegs als ein Factum vorauszusetzen nötig (ja als
ein solches gar nicht möglich); ... Sondern es ist eine bloße Idee der Vernunft, die
aber ihre unbezweifelbare (praktische) Realität hat: nämlich jeden Gesetzgeber zu
verbinden, daß er seine Gesetze so gebe, als sie aus dem vereinigten Willen eines
ganzen Volks haben entspringen können, und jeden Untertan, so fern er Bürger
sein will, so anzusehen, als ob er zu einem solchen Willen mit zusammen gestimmet
habe. Denn das ist der Probierstein der Rechtmäßigkeit eines jeden öffentlichen
Gesetzes.“ (Gemeinspruch, A249-250)

Soweit Kant sich auch in der politischen Philosophie einer legitimatorischen Schilderung der Staatsgründung aus einem Naturzustand heraus bedient, fallen die Kontraste schon deshalb weniger dramatisch aus, als die Leidenschaften von einer eben nicht nur instrumentellen Vernunft flankiert werden. So unterscheidet die Rechtslehre zwar noch den Naturzustand vom staatlichen Zustand, aber dieser vorstaatliche Zustand ist nicht ein latenter Kriegszu- 
stand, sondern ein bereits gesellschaftlicher Zustand mit privatrechtlich individuellem Besitz, dem nur die staatliche Legitimation fehlt (MdS-RL, $\mathbb{S} 43$ ). Das wird möglich, weil die nicht nur durch ihre Leidenschaften geprägten Bürger auch ohne Staat imstande sind, einen friedlich geregelten Zustand aufrecht zu erhalten. Auch ohne eine zentrale Zwangsgewalt sind sie in der Lage, einander als gleiche zu achten und anzuerkennen. Der Staat dient einer nachträglichen Legitimation und Stabilisierung des Besitzes, der bereits vor ihm moralisch ermöglicht wird. Entsprechend dieser reduzierten Funktion des Staats behalten die Bürger ihm gegenüber auch ihre moralische Kontrollfähigkeit.

Ebenso wie Hobbes will Kant nicht einfach nur eine philosophische Theorie des Staatsrechts vorlegen, sondern ein reales Staats- und Bürgerverständnis als vernünftig auszeichnen, genauer sowohl dafür normativ plädieren wie es in rekonstruktiver Absicht historisch beschreiben. Wiederum ist die Eigenständigkeit der Freiheit der Schlüssel für die entsprechende Alternative zu Hobbes. Denn zugestanden: eine "gesetzliche Verfassung nach Freiheitsprinzipien“ wird in der Praxis nicht anders möglich sein, als in einem Anfang „durch Gewalt, auf deren Zwang nachher das öffentliche Recht gegründet wird ..." (Frieden, B74). Und unstrittig, daß es in der Gesellschaft eines Systems von Rechten bedarf, als dem „Inbegriff von Bedingungen, unter denen die Willkür des einen mit der Willkür des anderen nach einem allgemeinen Gesetze der Freiheit zusammen vereinigt werden kann“ (MdS-RL, $\mathbb{S}$ B). Aber die Struktur und die Grenzen des Rechtssystems werden von einer moralischen Idee der gleichen Freiheit kontrolliert und damit der gesellschaftlich oder historisch willkürlichen Auslegung von Rechten entzogen.

Die Unabhängigkeit von Leidenschaften und Vernunft drückt sich wie in der Auslegung der einzelnen Rechte so auch im ganzen in dem Zweck aus, dem die Staatsbildung untersteht. Im Hobbesschen Vertrag suchen die Bürger ihre Lebensziele zu sichern, die sie anders als durch Unterwerfung nicht realisieren können. Der Zweck des Vertrags wie auch des staatlichen Rechts, ja vielleicht sogar der Gesellschaft, ist nichts anderes als die individuelle Lebenssicherheit. Die Kantschen Menschen benötigen zwar in ähnlicher Weise Schutzrechte voreinander, und die Rechte haben deshalb eine ähnlich nützliche Funktion zu erfüllen. Weil die Vernunft jedoch nicht dem obersten Zweck der Lebenssicherung untersteht, sondern von ihr unabhängig ist, drückt sich in allen Rechten und generell im Staat ein Vorrang der moralischen Gemeinsamkeit und Achtung aus. Der Staat ist auch, aber nicht vorrangig eine Institution der Lebensbewältigung, er ist vorrangig eine Institution der Anerkennung Gleicher.

„Der Begriff eines äußeren Rechts überhaupt geht gänzlich aus dem Begriffe der Freiheit im äußeren Verhältnisse der Menschen zu einander hervor und hat gar nichts mit dem Zwecke, den alle Menschen natürlicher Weise haben (der Absicht auf Glückseligkeit), und der Vorschrift der Mittel, dazu zu gelangen, zu tun ... 
Recht ist die Einschränkung der Freiheit eines jeden auf die Bedingung ihrer Zusammenstimmung mit der Freiheit von jedermann, in so fern diese nach einem allgemeinen Gesetze möglich ist; und das öffentliche Recht ist der Inbegriff der äußeren Gesetze, welche eine solche durchgängige Zusammenstimmung möglich machen." (Gemeinspruch, A234)

„In [der] Vereinigung [der drei Staatsgewalten] besteht das Heil des Staats ...; worunter man nicht das Wohl der Staatsbürger und ihre Glückseligkeit verstehen muß; denn die kann vielleicht (wie auch Rousseau behauptet) im Naturzustande, oder auch unter einer despotischen Regierung, viel behaglicher und erwünschter ausfallen: sondern den Zustand der größten Übereinstimmung der Verfassung mit Rechtsprinzipien versteht, als nach welchem zu streben uns die Vernunft durch einen kategorischen Imperativ verbindlich macht." (MdS-RL, A173)

Welche Folgerungen lassen sich aus dieser ersten Konfrontation ziehen? Was Hobbes und Kant verbindet: die je spezifische Auffassung von Moral und Politik beider, ließe sich nicht verstehen ohne das jeweilige Verhältnis, das beide der Moral und Politik zueinander beimessen. Ungeachtet der Differenz beider in der Art und Weise, wie sie Moral und Politik rationalisieren und gestalten wollen, ist doch klar, daß sich ihnen zufolge eine normative Theorie in beiden Bereichen, oder auch in einer Vorstellung von der Gesellschaft insgesamt, bewähren muß. Vereinfacht könnte man sagen, daß eine Moraltheorie politiktauglich oder eine Politiktheorie moralbewußt sein muß. Hobbes und Kant führen dazu zwei Szenarien vor, die gerade ihrer Gegensätzlichkeit wegen erhellend, und (wie eingangs vermutet) vielleicht sogar erschöpfend sind. Beide zeigen auf verschiedene Weise, wie sich eine politische Ordnung als moralische verstehen läßt oder wie sich Politik und Moral zueinander verhalten können.

Was Hobbes und Kant unterscheidet, läßt sich anhand der unterschiedlichen Vernunftauffassungen am leichtesten aufzählen. In einer Hinsicht ist klar, daß sich Kants Politikverständnis in der Gegenwart, bis in aktuellste Varianten einer, deliberativen Demokratie', als eher tauglich erwiesen hat als Hobbes' autoritärer Staat. Dennoch bleibt die Hobbessche Tradition lebendig - vor allem aus zwei Gründen heraus. Der erste und vielleicht wichtigste Grund: den Interpreten und Verteidigern von Kants Ethik ist es bisher nicht überzeugend gelungen, Kants kategorischen und darin ,stärker' als nur instrumentellen Vernunftbegriff selbst moralisch voraussetzungslos zu formulieren. Damit bleibt die Skepsis der Hobbesschen und Humeschen Tradition bestehen, wonach die praktische Vernunft nur eine instrumentelle Vernunft sein kann, die von psychologischen Voraussetzungen abhängig ist, um moralische und politische Normen zu rechtfertigen.

Zweitens materialisiert sich diese Skepis natürlich ebenso in Gestalt einer Opposition gegenüber der kantianisch, etwa diskursethisch verstandenen Demokratieauffassung, der gegenüber sich die heutigen Nachfolger des Hob- 
besianismus nicht auf den autoritären Staat, sondern, wie J. Buchanan oder D. Gauthier, auf eine minimalistisch-moralische Form des Wirtschaftsliberalismus stützen wollen. Die kantianische Vernunft gilt den modernen, häufig auch an Hume und Hayek orientierten Liberalen nur als eine besondere Form von Moral, gegen die sie ihr instrumentelles Freiheitsverständnis ins Feld führen. Im großen und ganzen ist der hobbesianische Neokontraktualismus allerdings weitgehend politikabstinent und verbleibt im Bereich der ethischen Grundlagendebatte, im Unterschied zu seinem großen Vorbild. ${ }^{7}$

Die kantianische Tradition der Ethik hat ihrerseits zwei Strategien verfolgt, um zusammen mit ihrem stärkeren Vernunftbegriff eine mehr als nur minimalmoralische, nämlich Gerechtigkeit umfassende Form des Liberalismus gegen die Kritiker zu verteidigen. Die erste Strategie ist vor allem mit dem Namen von J. Rawls verbunden und besteht darin, die bereits moralische Qualität des starken Vernunftbegriffs zuzugestehen, eine damit verbundene Kritik jedoch zurückzuweisen. Anhand eines kurzen Überblicks zu Rawls' Theorie soll diese Strategie gleich ausführlicher geschildert werden. Eine zweite Strategie hat sich im Neokantianismus der letzten Jahre entwickelt. Bei ihr geht es darum, Kants genuinen Vernunftanspruch zu retten und den moralischen Gehalt einer als universal bestimmten praktischen Vernunft als sowohl unausweichlich und notwendig, zugleich aber metaphysikfrei und damit allgemein akzeptabel auszuweisen. Der Geist der Kantschen Vernunftethik soll bewahrt, Kants Freiheitsmetaphysik hingegen umgangen werden. Besonders inspirativ wurde diese zweite Strategie in letzter Zeit vor allem von Rawls-Schülern wie C. Korsgaard und T. Scanlon entwickelt. ${ }^{8}$

\section{Rawls und die kantianische Vertragstheorie}

Ironischerweise hat Rawls gerade anhand seines epochalen Versuchs, die Vertragstheorie im kantianischen Sinn zu erneuern, nicht nur zum Entstehen des gegenwärtigen Neokantianismus, sondern auch zur Wiederentdeckung der Hobbesschen Vertragstheorie beigetragen. Indem er seine Prinzipien der Gerechtigkeit letztlich auf die moralischen Intuitionen durchschnittlicher Bürger westlicher Gesellschaften zurückführte, entzündete er auch Motive für eine anspruchsvollere, von diesen Intuitionen unabhängige Vernunftbegründung, die dann nur entweder in die bereits angekündigte Richtung des Hobbesschen Programms oder in die Richtung einer Rekonstruktion des star-

7 Gauthiers Neigung zu einem Liberalismus mit minimaler Moral ist aber offenkundig: s. Gauthier (1986) Kap. 11; (1997).

8 Die ausgesprochen fruchtbare angelsächsische Kant-Renaissance der letzten Jahre verdankt neue Impulse außerdem T. Hill, B. Herman, M. Baron, O. O’Neill und D. Guevara. 
ken Kantischen Vernunftbegriffs gehen kann. ${ }^{9}$ Bevor wir einen Blick auf diese Alternativen werfen, ist es nötig, Rawls' Theorie der Gerechtigkeit in ihrer Begründungsabsicht zu vergegenwärtigen.

Die Theorie der Gerechtigkeit kombiniert mehrere Begründungsideen, unter denen der Vertragsgedanke jedoch mindestens der Absicht nach im Zentrum steht - und zwar, wie Rawls betont, in der Version von Locke, Rousseau und Kant, nicht von Hobbes (TG, 27). Von einer Vertragstheorie spricht Rawls, weil er einen dem Naturzustand ähnlichen hypothetischen Zustand zum Kern eines neuen Begründungsarguments für politische Institutionen macht. Er nennt diesen Zustand schlicht ,Urzustand' und versucht mit ihm zweierlei zu erreichen. Erstens wird beabsichtigt, eine Wahl von Moralprinzipien unter vorrangig selbstinteressierten Akteuren zu modellieren. Dabei werden Prinzipien der rationalen Entscheidungstheorie einbezogen, um die Art dieser Wahl zu präzisieren und rational durchsichtig werden zu lassen. Die Referenz auf Locke, im Unterschied zu Hobbes, deutet jedoch bereits an, daß sich Rawls mit einer rein selbstinteressiert-rationalen Wahl nicht begnügen will.

Zusätzlich, zweitens, hat die Wahlsituation insofern eine dezidiert moralische Qualität, als sie durch bestimmte Restriktionen der Wahl einen Standpunkt der ,Fairness' oder der Unparteilichkeit wiederzugeben versucht. Zu den Restriktionen gehören insbesondere gegenseitiges Desinteresse und der berühmte "Schleier des Nichtwissens“ (TG, $\mathbb{S} 24)$, der den Wählenden das Wissen über ihre persönlichen Lebensumstände vorenthält und sie damit nicht nur zwingt, keine Ausnahmen im eigenen Interesse zu machen, sondern es erst ermöglicht, daß überhaupt ein Konsens in der Wahl von Prinzipien erreicht wird. Mehr noch: für risikoscheue Wähler soll es rational zwingend werden, mit der Entscheidung für das „Differenzprinzip“ zugunsten einer Form von sozialer Gerechtigkeit zu votieren, in der gegenüber den sozial schlechter Gestellten eine starke Rechtfertigungspflicht entsteht. Im Ergebnis wählen die selbstinteressiert-rationalen Entscheider damit eine die sozial Schwachen besonders berücksichtigende Gerechtigkeitsmoral.

Dieses zwar originelle, aber hybride Kernstück in Rawls' Aktualisierung des Vertragsgedankens brachte eine Reihe von Schwierigkeiten mit sich, die insgesamt dafür verantwortlich sind, daß man heute Rawls kaum noch als Vertragstheoretiker liest. Zunächst wollte Rawls die Gerechtigkeitstheorie als Bestandteil der rationalen Entscheidungstheorie verstanden wissen (TG, 33, 196), in Verbindung eben mit, wie er meinte, ,moralanalogen' und nicht selbst moralischen Restriktionsbedingungen der Wahl von Prinzipien. Im Gegensatz

9 Oder die Kants Ansatz irgendwie mit Aristoteles verbindet. Das scheint mir, wie ich noch zeigen will, bei Scanlon der Fall. 
zu einer verbreiteten Ansicht ist dieser Versuch nicht einfach zirkulär, denn die moralischen Randbedingungen der Wahl decken sich nicht inhaltlich mit den zwei Prinzipien der Gerechtigkeit, deren Unter- und Metaprinzipien, also den moralischen Folgerungen des gesamten Arguments. ${ }^{10}$ Allerdings hat sich die Aufmerksamkeit vieler immer mehr auf die moralischen Prämissen gerichtet sowie auf die damit verbundene Frage, inwieweit die moralischen und prudentiellen Bestandteile des Arguments miteinander harmonieren können.

Um solche Einwände zu berücksichtigen, hatte Rawls das Vertragsargument in ein weiteres Begründungskonzept eingebettet, wonach moralische Urteile dann als begründet gelten können, wenn sie untereinander kohärent sind und unsere wohlüberlegten „moralischen Intuitionen“ zum Ausdruck bringen, oder anders, wenn ein „Überlegungsgleichgewicht“ besteht (TG, \$9). Mit den moralischen Intuitionen werden nicht die mysteriösen Gegenstände eines speziellen ,moralischen Sinns' angesprochen, sondern die moralischen Überzeugungen des Alltags. Rawls säkularisiert gewissermaßen die religiös gesättigten moralischen Urteile der Naturrechtstradition, indem er sie für ethische Argumente als unhintergehbar postuliert - allerdings nicht in ihrer faktischen, sondern in ihrer, wohlüberlegten' Form. In den nötigen Prozess des Überlegens können verschiedenste Arten von Argumenten einbezogen werden, und dabei insbesondere prudentielle wie das der Wahl von Prinzipien in der geschilderten Ursituation. Das prudentielle Argument soll den moralischen Intuitionen gegenüber soweit klärend wirken, daß die Gesamtüberlegung - gegeben unsere bereits vorhandenen moralischen Intuitionen - so weitgehend zwingend wie möglich, eben „vernünftig“ (TG, 35-37), werden sollte. ${ }^{11}$ Rawls nannte das Argument ursprünglich ziemlich optimistisch ein „streng deduktives Argument“ zugunsten der Prinzipien der Gerechtigkeit (TG, 143).

Eine Reihe von Problemen erschwerten jedoch diese Erwartung, die sich in verschiedenen Arten von Einwänden ausdrückten. Solche Einwände richteten sich typischerweise auf Rawls' spezielle Inanspruchnahme der rationalen Entscheidungstheorie sowie auf das klassische Problem der Vertragstheorie, inwieweit fiktive Szenarien trotz ihrer Irrealität Argumentationskraft haben

10 S. auch die ausführlichere Kommentierung des Vertragsarguments durch Maus (1998), die auch Vergleiche mit der älteren Vertragstradition anstellt.

11 S. auch Wolff (1977) Kap. 1. „Vernünftig“ (reasonable) wird von Rawls 1971 als gleichbedeutend mit "rechtfertigbar" eingeführt und von vornherein mit einer moralinternen Art des Rechtfertigens identifiziert. „Vernünftig erscheint die Annahme, daß die Menschen im Urzustand gleich seien.“ (TG, 36) - Die deutsche Übersetzung von 1975 lässt bekanntlich die terminologische Differenz von „reasonable“ und „rational“ (nutzenoptimal) im Text nicht sichtbar werden. 
können. ${ }^{12}$ Die meines Erachtens interessantere Art von Einwänden problematisiert hingegen die Art und Weise, wie Rawls glaubte, prudentielle und moralische Teilargumente miteinander verbinden zu können. Dazu gehört erstens eine Kritik der Dominanz des Prudentiellen, wie sie sich im Optimismus der entscheidungstheoretischen Rationalität der Theorie der Gerechtigkeit ausdrückt, bzw. Verweise auf die Unvereinbarkeit des prudentiellen Vorrangs mit den moralischen Rahmenbedingungen. Und zweitens eine Kritik generell des Rekurses auf die moralischen Intuitionen und die damit eingeräumte Ausgangsbasis in der Kontingenz einer bestimmten moralischen Kultur, deren historische Diagnose ja durchaus umstritten ist. Beide Arten von Kritik erscheinen umso gewichtiger, als Rawls mit den Anspruch einer kantianischen Position aufgetreten ist (s. TG, $\mathbb{S} 40$ ) und Kant selbst am Vorrang der Moral vor der Klugheit sowie am absoluten Geltungsanspruch der Moral keinen Zweifel gelassen hat (GMS, 2. Abschnitt).

In Anschluß an ihre erste Fassung von 1971 hat Rawls seine Theorie auf vielfältige Weise umgestaltet und erweitert. Das Ausmaß dieser Revisionen im Verlauf von fast 30 Jahren ist zu komplex, als daß sich die Motive klar und einfach benennen ließen, die Rawls zu seinen revidierten Positionen in Political Liberalism (1993) und Justice as Fairness: A Restatement (2001) geführt haben. Zusammenfassend und speziell unter dem Aspekt des Begründens gilt jedoch, daß er die Kritik an der Kombination von Prudentiellem und Moralischem damit beantwortet, die Moral klar vorzuordnen, und daß er auf die Kritik an der begründungstheoretischen Schwäche des Ausgangs von den moralischen Intuitionen mit einem veränderten Verständnis seiner Theorie reagiert. War die Theorie der Gerechtigkeit als umfassender moralphilosophischer Traktat gemeint, in dem auch noch die individuelle Moral ihren Platz hatte (T9, $\mathbb{S}$ 18-19), so wird die spätere Theorie dezidiert als eine politische vertreten (JF, $\mathbb{S} \mathbb{S} 1,9,11,54)$. Diese Wendung mag verschiedene Motive haben, sie kommt jedoch auch dem Einwand des Sichberufens auf die moralischen Intuitionen entgegen. Im Grunde ist jetzt das gegenseitige Respektieren der moralischen Ansichten die Basis, ohne daß diese Ansichten ihrerseits mit objektivem Anspruch verbunden wären. Die Basis ist ein moralisches Argumentieren gegeneinander (so bereits TG, $\mathbb{S} 87$, bes. 630).

Rawls teilt nämlich die Skepsis der empiristischen Tradition am starken Vernunftbegriff Kants. Obwohl er in der inhaltlich von ihm vertretenen Moral immer den „Vorrang des Rechten vor dem Guten“, also die individuellen Rechtsansprüche vor dem rechteunsensiblen Erfüllen von Interessen,

12 Hypothetische Verträge können nur hypothetisch binden, so Dworkin (1977). Diese Art von Einwand geht zurück auf Hume, C. 
betont (TG, 42f., 48ff.; JF, 81), beruft er sich zugunsten dieses Vorrangs nie auf den Kantischen Vorrang der kategorisch geltenden Vernunft vor der hypothetischen der Klugheit. Das politische Verständnis der eigenen Theorie wird explizit gegen ein metaphysisches, oder ein philosophisches, das keine allgemeine Geltung erlangen kann, abgesetzt (JF, $\mathbb{S} 1$ ). Ein Rekurs auf die Philosophie Kants im Rahmen einer politischen Moral müsste, als Grundlage von staatlicher Macht, zu Repression und Intoleranz führen und ist deshalb mit einem Liberalismus, wie er Rawls vorschwebt, nicht vereinbar (JF, 34). Damit kommt Rawls der empiristischen Tradition aber möglicherweise weiter entgegen, als ihm selbst lieb sein kann.

Anhaltende Skepsis muß sich gegen den Status der Rawlsschen Verwendung des Prädikats „vernünftig“ richten, einen Anspruch, der eingestandenermaßen moralisch, wenn auch minimalistisch moralisch sein soll. Ob die didaktisch gemeinte Schilderung der Ursituation aber gerade geeignet ist, den richtigen moralischen Minimalismus aus den verbreiteten moralischen Ansichten herauszuheben, lässt sich bezweifeln, in Gestalt anhaltender moralischer Opposition sogar begründet bezweifeln. Ein Rest des Kantischen Apriorismus kommt versteckt in der Nebenbemerkung von Rawls zum Ausdruck, ,vernünftig“ heiße „,intrinsisch vernünftig“ (JF, 26, 30f.). Wenn die um eine angemessene moralische Gestaltung ihrer Gesellschaft ringenden Bürger erfolgreich sein wollen, so müssen sie sich selbst als vernünftig verstehen, sich gegenseitig als Quellen von Wert anerkennen. Im Kontrast zu Rawls' Abgrenzung gegenüber jeder Metaphysik ist das eine vieldeutige und dadurch besonders interessante Äußerung. Man kann sie sowohl zum Anlaß dafür nehmen, im Geist des Rawlsschen Programms den starken Vernunftbegriff metaphysikfrei zu präzisieren, wie, in Übereinstimmung mit Rawls' Skepsis, auf das Ausgehen von moralischen Intuitionen ganz zu verzichten.

\section{Scanlon und das Reich der Gründe}

Scanlon verfolgt die erste Alternative. Wenn wir uns an die bisher geschilderten Begründungsalternativen und an die mit ihnen verbundenen Begriffe halten, erscheint die Position eines ,moralischen Kontraktualismus' genau genommen unmöglich. Das Attribut des Moralischen soll ja nicht das Handlungsziel, also die Rechtfertigung moralischer Normen, sondern die Voraussetzungen bezeichnen. In der Hobbesschen Tradition sind die Voraussetzungen für Übereinkünfte moralfrei, der rationale Ausweis eines Übereinkommens führt dementsprechend einen klaren rationalen Zwang für diejenigen mit sich, die die moralfreien Voraussetzungen teilen. Der rationale Zwang durch Übereinkommen korrespondiert mit moralfreien Voraussetzungen. Umgekehrt hat Rawls' Ursituation nichts mit einem Vertrag oder Übereinkommen gemein. Der Schleier des Nichtwissens reduziert eine beliebige Menge von 
Entscheidern auf einen einzigen, der stellvertretend für alle, epistemisch mit ihm Gleichgestellten, entscheidet. Moralische Voraussetzungen schränken die praktische Funktion von Übereinkommen gerade ein. Der ,Vertrag' ist von einer moralfreien Übereinkunft in den Interessen abhängig, so daß ein ,moralischer Vertrag' bestenfalls gegenständlich, als das gemeinsame Festlegen von moralischen Normen verstanden werden könnte.

Weder Kant noch Rawls können in ihren Texten den Vertragsbegriff im eigentlichen Sinn verwenden. Wie bereits geschildert, benutzt Kant die Rede vom Vertrag, um eine zwar moralisch gegründete, aber dennoch nicht vollständig moralische, sondern ,äußere', nämlich rechtliche Anerkennung auszudrücken. Die Kantische Rechtssphäre steht gleichsam in der Mitte zwischen den genuin moralischen Verhältnissen und den allein auf Zwang beruhenden. Rawls vermeidet den Vertrag als konstruktiven Begriff und soweit er seine Theorie in die Vertragstradition einreiht, ist das nur indirekt und uneigentlich gemeint. Beide Philosophen, in Übereinstimmung auch mit K.O. Apel und J. Habermas, die ihre Positionen unter den Etiketten ,Konsenstheorie“ und ,Diskursethik' vorgestellt haben, würden die Unvereinbarkeit einer konstruktiven Rolle von Gründen und Vertrag betonen. Diese Unvereinbarkeit geht soweit, daß häufig selbst die konstruktive Rede vom Konsens bezweifelt wird. Wozu Konsens, wenn allein die Gründe darüber entscheiden, was konsentierbar ist? ${ }^{13}$

Die Originalität von Scanlons moralischem Kontraktualismus besteht darin, daß er diesen Titel sowohl im eigentlichen Sinn versteht, wie ihn auf gewisse Weise zurecht in Anspruch nimmt. Wie Kant, Rawls, Apel und Habermas sieht Scanlon praktische Gründe nicht aus Interessen und Wünschen entspringen, sondern billigt ihnen Eigenständigkeit zu. Im Unterschied zu diesen Philosophen entwickelt er eine Auffassung von praktischen Gründen, die Gründe weder in metaphysischen Prämissen, moralischen Intuitionen oder universalen Kommunikationsnormen fundiert. Weiter, und wichtiger, bricht er außerdem mit einer implizit oder explizit üblichen Annahme, wonach moralische Gründe primär generelle, oder genauer, akteursneutrale Gründe sind. Akteursneutrale Gründe gelten für alle Akteure gleich und sind eng mit dem Konsequentialismus verbunden. Seiner Meinung nach sind praktische Gründe wesentlich akteursrelative Gründe, Gründe, die nur für denjenigen gelten, der sie hat. Dies setzt eine motivationale und nicht kognitive Form von Gründen voraus. Auf diese Weise wird verständlich, inwiefern das Begründen mit- und gegeneinander zugleich ein gründeorientiertes wie ein konstruktives

13 Diesen Einwand hat in der deutschsprachigen Diskussion vor allem E. Tugendhat vorgebracht; s. bes. Tugendhat (1993) Vorl. 8. 
Geschehen sein kann, in dem nicht allein durch das Gegebensein von Gründen alle Resultate von vornherein entschieden sind. ${ }^{14}$

Der Natur seines Ansatzes gemäß muß Scanlon seine Theorie nach zwei Seiten verteidigen. Auf der einen Seite gegenüber der empiristischen Tradition, die Gründe nur in Wünschen sieht und ein Rechtfertigen der Moral mit einem moralexternen Standpunkt verbinden will, wie etwa dem Naturzustand. Dieser Tradition gegenüber will Scanlon zeigen, daß moralische Gründe solche sind, die jeweils andere „nicht vernünftigerweise zurückweisen können“. Offensichtlich inspiriert von Rawls' Begründen als einem Ausweisen gegenüber anderen, transformiert Scanlon außerdem Rawls' Rekurs auf moralische Intuitionen in einen Rekurs auf Gründe. Während Rawls', moralische Intuitionen' psychische Zustände mit begrenzter Rationalisierbarkeit bleiben, sind nach Scanlon dezidiert moralische Gründe das repräsentative Feld, auf dem sich moralische Debatten alltäglicher wie philosophischer Art zutragen. ${ }^{15}$ Gründe und die mit ihnen verbundenen Wertgesichtspunkte können nicht auf Wünsche reduziert werden, während Wünsche Gründe benötigen, um ihre Motivationsfunktion zu erfüllen.

Gründe suchend können wir uns nicht außerhalb des Reichs der Gründe stellen: das mag man vielleicht noch eingestehen - aber muß dieses ,Reich ${ }^{6}$ gerade eines der ,Zwecke', also eines der moralischen Gründe sein? Und selbst wenn, in welchem inhaltlichen Sinn von Moral? Sind dabei Amoralismus und Relativismus nicht auch möglich? Scanlons Antwort auf diese Fragen besteht in einer Analyse sozialer Beziehungen, in denen der Wert der Beziehung eng verbunden ist mit der Anforderung einer über Gründe ausgewiesenen gegenseitigen Anerkennung. ${ }^{16}$ Scanlons Absicht ist es zu zeigen, daß die persönlichen Beziehungen auch einen Modellfall für die sozialen Beziehungen im allgemeinen abgeben, insofern sie neben ihrem Vorrang an persönlicher Bedeutung auch ein Element der universellen Rechtfertigbarkeit von Beziehungen, ja von Lebensgütern im allgemeinen enthalten. ${ }^{17}$ Amoralisten und Relativisten sind spezielle Arten von Skeptikern, denen die inhärenten Qualitäten der sozialen Beziehungen verborgen bleiben. Ihnen gegenüber ist es

14 S. auch (Ridge) 2001. Auf den wichtigen Unterschied zwischen akteursneutralen und -relativen Gründen hat als einer der ersten T. Nagel (1987, Kap. 9) hingewiesen. Diese Unterscheidung ist dementsprechend nicht zu verwechseln mit derjenigen zwischen ,objektiven' und ,subjektiven' Gründen, so daß sich aus der Betonung der akteursrelativen Gründe nicht folgern läßt, es werde ein mit dem Nonkognitivismus vergleichbares Konzept der subjektiven Gründe vorgeschlagen. Akteursrelative Gründe können ebenso objektiv sein wie akteursneutrale. Scanlon nennt letztere ,unpersönliche' Gründe; s. Scanlon (1998) 218-223.

15 Scanlon (1998) Kap. 1.

16 Ebd. 162.

17 Ebd. 162-168. 
hinreichend, wenn auch im konkreten Fall nicht immer erfolgreich, auf diese Qualitäten hinzuweisen. ${ }^{18}$

Scanlons Ausführungen an diesem zentralen Punkt scheinen mir unentschieden und in jedem Fall zu knapp. Sie schwanken zwischen, wie man sagen könnte, einer ,kantianischen' und einer , aristotelischen' Rekonstruktion der Freundschaft. Damit sind zwei Alternativen gemeint, wie die Beziehung zu einer besonderen Person mit den Rechtfertigungsbeziehungen verbunden sein kann. In der kantianisch verstandenen Freundschaft ist die persönliche Beziehung nur eine emotionale Zugabe zu den Rechtfertigungsbeziehungen, die den Freund wie alle Menschen erfassen. Pflichtenkonflikte, die den Freund und Fremde betreffen, werden unparteilich geschlichtet. Wird die Freundschaft jedoch, wie von Scanlon, als ein moralisches Gut angesehen, dann bleibt unklar, warum die Pflichtenkonflikte anders als immer zugunsten der Freunde gelöst werden sollen. Scanlon behauptet, daß die unparteiliche Moral der Freundschaft auch bereits inhärent sei. ${ }^{19}$ Wie das möglich ist, wäre allerdings erst zu zeigen. Das aussergewöhnliche Gut der Freundschaft zu bestimmten Personen legt der universellen Moral eher Grenzen auf oder macht sie sogar unmöglich.

An diesem Abweichen gegenüber dem üblichen kantianischen Muster deutet sich bereits auch die zweite Verteidigungslinie an, die Scanlon einnimmt. Nicht nur Freundschaften, sondern auch die meisten anderen sozialen Beziehungen sind im wesentlichen keine ,diskursiven', sondern emotionale Beziehungen, und die Diskursethik muß deshalb die sozialen Beziehungen einseitig in ein überrationales Muster pressen. Alle sozialen Beziehungen, nicht nur die persönlichen, umfassen einen persönlichen Standpunkt, denjenigen des Akteurs, und die Gründe im Handeln gegen andere müssen von diesem Standpunkt aus entwickelt werden. Im Gegensatz zu sowohl den Konsequentialisten wie den meisten Kantianern ist Scanlon gleichsam ein ,Personalist ${ }^{6}$, nach dem moralische Gründe im Rahmen der moralischen Güter je einzelner Personen entspringen und erst nachträglich mit den Gütern bzw. Gründen der anderen Personen gleichsam verrechnet werden müssen. Die Ähnlichkeit mit dem üblichen Interessenkontraktualismus ist offensichtlich - der Unterschied liegt jedoch darin, daß der Übergang von der individuellen auf die überindividuelle, gesamtgesellschaftliche Ebene anhand von moralischen Gründen ermöglicht werden soll, die in den moralischen Gütern als akteursrelative oder persönliche Gründe allen einigermaßen gleich zugänglich sind.

Terminologisch markiert Scanlon den Übergang von der nur individuellen zur auch überindividuellen Ebene mit dem Begriff der „allgemeinen“ (generic)

18 Ebd. 148, 158, 353.

19 Ebd. 165. 
Gründe. ${ }^{20} \mathrm{Zu}$ allgemeinen Gründen werden Gründe dann, wenn sie auf eine an Kant erinnernde Weise universalisiert werden: dem Grund korrespondiert ein Prinzip, das in der Gesellschaft allgemein befolgt wird. Anders als bei Kant ist mit Prinzipien aber kein singuläres Universalisierungskriterium verbunden, weder eines des Widerspruchs noch ein konsequentialistisches. (Den Konsequentialismus insbesondere lehnt Scanlon unter anderem mit dem originellen Argument ab, daß das individuelle Wohl zu vage sei, um isoliert als Grund zu dienen. ${ }^{21}$ ) Vielmehr sollen allgemeine Gründe von vornherein eine eigenständige Bedeutung haben, stehen allerdings immer in Konkurrenz sowohl untereinander wie mit persönlichen Gründen innerhalb eines ,Holismus der Gründe‘.22 Obwohl Scanlon Rawls' Idee der Fairness als einer Grundeigenschaft der heutigen Moral teilt, hält er eine didaktische Konstruktion wie die Ursituation für unnötig. Umgekehrt bleibt aber in seiner Darstellung offen, wieso und in welchem Ausmaß moralische Gründe als allgemeine Gründe vorgebracht werden müssen.

Setzt man wie Scanlon moralische Gründe mit personalen Gründen gleich, ist natürlich ein übliches Argument ausgeschlossen, wonach Gründe deshalb per se allgemeine Gründe sind, weil es eben Gründe sind. Gründe mögen als theoretische allgemein sein, als moralische beginnen sie vom persönlichen Standpunkt aus. Soll der moralische Kontraktualismus deshalb nicht in eine beliebige Menge von mehr oder weniger stark gestaffelten moralischen Teilgemeinschaften, mit entsprechend gestaffelten moralischen Pflichten, zerfallen, so muß genauer gezeigt werden, inwiefern die Idee einer unpersönlichen Moral in den persönlichen Moralen enthalten oder vorweggenommen ist.

Die Grundidee, der Scanlon zu folgen scheint, die er aber nicht wirklich ausarbeitet, könnte diese sein: tiefe soziale Beziehungen müssen auch Rechtfertigungsbeziehungen sein, im Sinn eines mindestens potentiellen Rechtfertigens gegenüber diesen anderen; und in dem Rechtfertigen gegenüber einzelnen anderen ergibt sich notwendigerweise eine Abstraktion von ihnen, der Rechtfertigende wird in dieser seiner Rolle gezwungen, seine persönlichen Gründe in allgemeine zu transformieren. Für diese Idee spricht vor allem die allgemeine Gültigkeit der theoretischen Vernunft und ihr notwendiger Zusammenhang mit der praktischen. Kant hat einen solchen Zusammenhang in der Rede vom moralischen ,Gesetz' ebenfalls postuliert, aber nie genauer nachgewiesen. Soweit bleibt also nur der Hinweis auf die Existenz der theoretischen Vernunft als Beleg der Vermutung, daß die Moral ebenso universal sein muß.

20 Ebd. 204.

21 Ebd. 133.

22 Ebd. 214-218. 


\section{Die deskriptive Rolle des Vertrags}

Die Begründungsansätze von Rawls und Scanlon sind in dem Sinn stark normativ, daß empirische Überlegungen zur Moral oder zur Gesellschaft bestenfalls eine restringierende Funktion spielen. Selbst Rawls' ausführliches Bemühen um die Stabilitätsbedingungen einer gerechten Gesellschaft (TG, Teil 3) modifiziert nur nachträglich die vorweg und weitgehend ideal gewonnene Grundstruktur dieser Gesellschaft. Beide, insbesondere auch Scanlon, betrachten das Begründen als ein der Gesellschaft gegenüber ideales Argumentationsspiel, in dem unter sozial gesehen idealen Bedingungen Argumente ausgetauscht werden, um Überzeugungen herzustellen. Der Begriff des Vertrags kann in einem solchen Spiel rein argumentativ benutzt werden und bleibt dann von empirischen Voraussetzungen weitgehend frei. Wird die Prämisse hingegen verworfen, wonach wir uns unausweichlich in einem bereits moralisch voreingenommenen Argumentationsspiel befinden, so werden die empirischen Voraussetzungen, die in der Idee eines Vertrags unter vielen Individuen prinzipiell enthalten sind, sofort wieder bedeutsam. Bevor ich auf den hobbesianischen Neokontraktualismus genauer eingehe, möchte ich eine Überlegung dazu anstellen, in welchem Ausmaß der Vertragsgedanke in dieser Tradition notwendig empirisch ist.

Hobbes' Vertragsargument etwa ist zweifellos in Hinblick auf die psychologischen und sozialen Annahmen über den Naturzustand mit empirischen Hypothesen über das Verhalten durchschnittlicher Menschen verbunden. Die Relevanz dieser Hypothesen für das Argument, auch wenn es sich als normatives Argument versteht, wird nicht dadurch neutralisiert, daß der Naturzustand als hypothetischer gemeint ist: damit der hypothetische Zustand praktisch relevant ist, muß er in der realen Welt relevant sein, und dafür können nur die empirischen Voraussetzungen einstehen. Es wäre deshalb ein grundsätzliches Mißverständnis, wollte man aus dem hypothetischen Status der moralfreien Vertragsargumente folgern, die Gültigkeit und Relevanz der in ihnen in Anspruch genommenen empirischen Hypothesen sei belanglos. Mangels vorausgesetzter Werte oder Normen kann ein solches Argument vielmehr gerade einzig aufgrund des empirischen Gehalts seiner Prämissen überzeugen.

Um der großen Bedeutung der empirischen Prämissen in nicht-moralischen Vertragsargumenten gerecht zu werden, scheint es hilfreich, eine häufig getroffene Unterscheidung zweier Typen von Kontraktualismus als miteinander verbunden, genauer als zwei Enden eines Kontinuums zu sehen, und nicht als einander ausschließend. Damit meine ich die Unterscheidung zwischen einem ,rechtfertigenden' und einem ,explanatorischen' Kontraktualismus. ${ }^{23}$ Daß

23 S. Schmidt (2000) $\$ 7$. 
sich diese beiden Formen des Kontraktualismus nicht völlig voneinander trennen lassen, ist auf der einen Seite dadurch bedingt, daß ein naturalistisches Rechtfertigen nicht ohne empirische Annahmen möglich ist, auf der anderen Seite dadurch, daß jedes Sozialverhalten nur unter Einbezug rationaler Gesichtspunkte erklärt werden kann.

Meiner These der gerade empirischen Signifikanz von nicht-moralischen Vertragsargumenten könnte entgegengehalten werden, daß sie doch bereits an dem Eingeständnis scheitert, daß ein realer Vertrag unter einer großen Zahl von Individuen nie möglich ist. Die Rede vom Vertrag, besonders deutlich bei Hobbes, ist tatsächlich in dem Sinn doppeldeutig, daß sie sowohl ein normatives Argument der Interessenübereinkunft wie, davon unabhängig (wenn auch daran geknüpft), einen Akt der normativen Selbstbindung bezeichnen kann. ${ }^{24}$ Mangels tatsächlichem Vertrag kommt nur die erste Alternative in Frage: der Vertrag kann nur für ein Argument der Interessenkonvergenz stehen, das den realen Individuen zugeschrieben werden kann, ohne daß sie selbst die Logik dieses Arguments kennen oder explizit akzeptieren müssen. Allerdings müssen die Prämissen des Arguments auf sie in irgendeiner Weise zutreffen. Das Ausmaß dieses Zutreffens kann variieren. Um dem gerecht zu werden, könnte man zwischen ,schwach-deskriptiven " und stark-deskriptiven "Versionen eines als solchen rechtfertigenden Kontraktualismus unterscheiden.

Wie man die bekannten Autoren dieser Unterscheidung zuordnet, ist teilweise der Interpretation überlassen. Hobbes selbst könnte man in jede der beiden Kategorien einordnen. Hobbesianer wie Gauthier, die sich auf spieltheoretische Argumente und das ökonomische Modell des Handelns stützen, sind eher stark-deskriptiv, solche wie Stemmer, die den Zweck der Vertragstheorie in einem gesellschaftsfernen Argumentieren unter Egoisten sehen, eher als schwach-deskriptiv. Rawls könnte man wegen seiner Annahme bestimmter Grundgüter schwach-deskriptiv nennen. Daß nicht alle Moralphilosophen in diese Kategorien fallen, liegt an der Verbindung dieser Kategorien mit dem Naturalismus. Moralische Kontraktualisten, wie insbesondere Scanlon, bestreiten die Möglichkeit von rein deskriptiven Prämissen in moralischen Argumenten, wie immer schwach diese ausfallen. ${ }^{25}$ Die Verwendung von empirischen Annahmen in Prämissen darf nicht mit dem Berücksichtigen empirischer Realisierungsbedingungen verwechselt werden. Letzteres gestehen natürlich mit Kant auch alle Kantianer zu.

Offensichtlich besteht eine große Nähe zwischen dem stark-deskriptiven und dem explanatorischen Kontraktualismus. Diese Nähe ist besonders greifbar in der Position von D. Hume. Hume hat deutlicher als seine sozialwissen-

24 S. Hampton (1986) Kap. 9; Gauthier (1988).

25 S. Scanlon (1998) Kap. 3. 
schaftlichen Nachfolger heute am Rechtfertigungsanspruch festgehalten, auch wenn er ihn zugleich in die Form einer sozialwissenschaftlichen Erklärung brachte. Hume hat bekanntlich die Idee des normativen Vertrags vehement kritisiert (C). Rechtfertigen und Erklären tiefer miteinander zu verbinden, ist allerdings nur so möglich, daß die eine Kategorie mit Gründen in die andere überführt wird. Gerade das hat Hume getan, insofern er in nahezu Hegelianischer Manier die Idee eines expliziten Vertrags an den Begründungsspielen der Philosophen vorbei in einen impliziten Konsens bzw. in die empirisch studierbare evolutionäre Konventionenbildung transformiert hat (T, III.ii). Nicht der Konsens erklärt politische Einigungen, sondern die Interessen der Akteure erklären den Konsens. Moralische Normen und politische Institutionen entstehen in einem nur halb bewußten und längerfristigen historischen Prozess, und sie sind dann vernünftig, wenn sie angemessener Ausdruck der verbreiteten Interessen sind.

Weil nach Hume ein Begründen der Moral eine solche Form des erklärenden Rationalisierens von moralischen Konventionen annimmt, ist es informativer, von einem moralischen ,Konventionalismus' zu reden. Allseits nützliche Konventionen ersetzen die konflikthaft auszuhandelnden Normen. ${ }^{26}$ Auf den ersten Blick mag vielen Humes Lösung als eine geschickte Mischung von sozialem Realismus und philosophischer Bescheidenheit erscheinen. Ist es nicht tatsächlich reichlich weltfremd, der realen Gesellschaft gegenüber einen Begründungsstandpunkt zu konstruieren, von dem her sie sich, möglicherweise in toto, ihre Gesetze geben soll, seien es moralische oder rechtliche? So müssen die philosophischen Begründungen jedoch nicht verstanden werden. Vielmehr können sie ideale Vorwegnahmen von, zugleich aber auch Initiativen für, die Reflexionen der Gesellschaftsmitglieder selbst sein. Hume war gegenüber der Philosophie und ähnlich auch gegenüber der sozialen Aufklärung ziemlich skeptisch eingestellt. Der Konventionalismus drückt diese Einstellung als eine reale politische Haltung aus. Die Humesche, Gerechtigkeit' ist vorrangig an sozialer Sicherheit interessiert und wiederholt damit die unerfreulichsten Aspekte des hobbesianischen autoritären Staats.

Explizite Plädoyers dafür, den rechtfertigenden Kontraktualismus durch eine humeanische Sozialtheorie zu ersetzen, werden selbst von denen, die in dieser Tradition arbeiten, kaum je vorgebracht. ${ }^{27}$ Das wäre umso nötiger, als der Konventionalismus keineswegs die unstrittig empirisch fruchtbare

26 Spieltheoretisch entsprächen dem die Koordinationsspiele; s. Schmidt (2000) $\mathbb{S} 25-27$, sowie als Klassiker Lewis (1969). Hume hatte aber einen weiteren Konventionenbegriff.

27 Eine Ausnahme ist Binmore (1994), im weiteren Sinn auch Hayek (1982). Der getreueste Humeaner in der gegenwärtigen Moraphilosophie ist vermutlich G. Harman: s. Harman (1988); (1996). Zur Kritik s. Stemmer (2000) 202-209. 
Theorie ist, als die er von seinen Anhängern gepriesen wird. Die empirischen Studien der ,Humeaner' sind in der Regel so weitgehend von ihrem speziellen Rationalitätsbegriff imprägniert, daß sie sich zur Selbstbestätigung meist besser eignen als zur Selbstkorrektur. Und klarerweise kann das instrumentelle Rationalitätsverständnis nicht selbst empirisch evaluiert werden.

Mit welchen Argumenten kann man sich zwischen schwach- oder starkdeskriptiv rechtfertigendem Kontraktualismus bzw. dem Konventionalismus entscheiden? In einer solchen Entscheidung kommt eine Ansicht zum Verhältnis von individuellem Standpunkt und Gesellschaft zum Ausdruck, sowie in Verbindung damit wohl auch von Moral und Politik. Wie wir anhand von Stemmers Ansatz gleich sehen werden, bleibt eine hochidealisierte individuelle Version des Kontraktualismus problematisch, wenn sie sich ihrer Rückbindung an die gesellschaftliche Realisierbarkeit nicht mehr versichert. Umgekehrt, wie sich anhand von Gauthiers Version sehen lässt, besteht eine Schwäche der eher gesellschaftlich ansetzenden Theorie darin, die notorische Lücke des ,trittbrettfahrenden Egoisten' nicht befriedigend schließen zu können. Nur diejenige Version des Kontraktualismus wäre erfolgreich, in der der repräsentative individuelle Standpunkt sowie die gesamte Gesellschaft als gleichgewichtige Teile eines Ganzen integriert wären. Bereits Hobbes war sich dieses Problems bewußt, und die heutigen Neokontraktualisten knüpfen meist an derselben Stelle an, an der Hobbes das Problem ad acta zu legen versucht hat: an den kritischen Einwänden gegen die gesellschaftlichen Normen in Gestalt des ,Foole‘ im XV. Kapitel des Leviathan.

\section{Hobbes redivivus}

„The Foole hath sayd in his heart, there is no such thing as Justice; and sometimes also with his tongue; seriously alleaging, that every mans conservation, and contentment, being committed to his own care, there could be no reason, why every man might not do what he thought conduced thereunto: and therefore also to make, or not make; keep, or not keep Convenants, was not against Reason, when it conduced to ones benefit." (Lev. XV, 4)

Der Foole widerspricht dem dritten natürlichen Gesetz, wonach Verträge nicht nur eingegangen, sondern auch erfüllt werden müssen (Lev. XV, 1). Dessen Formulierung ist mit dem begründenden Zusatz versehen, daß wir in dem Fall -, die Verträge wären nicht mehr als „empty words „- „... are still in the condition of Warre. "Das Einhalten der Verträge ist also ebenso wichtig wie ihr Zustandekommen. In dieser Gleichsetzung ist jedoch ein Rückschluß angelegt, aus dem die neueren Diskussionen zur Vertragstheorie ihren Zündstoff beziehen. Hobbes weist zwar eindringlich darauf hin, daß der Foole seine Einwände nicht im Naturzustand, sondern bereits unter den 
Sanktionsbedingungen des Friedenszustands vorbringt und dementsprechend dem Risiko dieser Sanktionen ausgesetzt ist. Die Gefahr des Rückfalls in die „condition of Warre“ verdeutlicht aber nur, daß die Einstellung des Fooles so nicht begrenzt werden kann und einen ernsthaften Zweifel aufwirft, ob ein verbindlicher Vertragsschluß überhaupt zustandekommen wird. Wie, könnte man fragen, kann sich Hobbes' Vertragsargument bewähren, wenn es nicht nur kollektiv-, sondern individuell-rational betrachtet wird?

Alle systematischen Versuche, eine hobbesianische Begründung von sozialen Normen zu geben, müssen sich deshalb dem Problem stellen, die gesamte Begründung an den Foole zu adressieren, seine Einwände können nicht auf ein nachträgliches Motivationsproblem begrenzt werden. ${ }^{28}$ Daneben wird eine hobbesianische Begründung immer dadurch ausgezeichnet sein, daß sie mit einer grundsätzlichen Differenz von Selbstinteresse und Moral rechnet und die Begründung unausweichlich ein Element der sozialen Sanktionen umfassen muß. Allerdings stehen für den logischen Ort der sozialen Sanktionen gleichsam zwei Systeme zur Verfügung: die Moral oder die Politik bzw. das Recht. Beide unterscheiden sich insofern, als das Recht den Individuen relativ gesehen äußerlich bleibt und es etwa nicht von vornherein widersprüchlich erscheint, das Befolgen von Rechtsnormen an Motive des Selbstinteresses zu binden. Korrespondierend mit diesem Unterschied ist das Recht notwendig mit einem expliziten Straf- und Sanktionsapparat verbunden, die Moral nicht unbedingt. Eine hobbesianische Begründung würde also, diesen Unterschied gegeben, naheliegenderweise als politisch-rechtliche Begründung entwickelt, nach dem Vorbild von Hobbes eigener Argumentation.

Bei den im weiteren Sinn als argumentative Rekonstruktionen von Hobbes' Vertragsargument angelegten Studien von J. Hampton (1986) und G. Kavka (1986) ist das der Fall. Beide Studien sind insofern interessant, als sich an ihnen zeigt, inwiefern Hobbes' eigener Gedankengang auch durch weitestgehende Präzisierungversuche, wie sie heute die Spieltheorie zur Verfügung stellt, dann nicht mehr stimmig nachvollzogen werden kann, wenn eben der individuelle Begründungsanspruch von vornherein als dominant in den Mittelpunkt rückt. Hampton und Kavka gehen durchaus verschiedene Wege. Hampton verwirft Hobbes' genuines Argument und versucht ein ihm möglichst nahe stehendes zu konstruieren, das sich dadurch auszeichnet, daß es die Macht des Souveräns abschwächt und einen Präferenzenwandel vorsieht. ${ }^{29}$ Auf diese Weise soll der sonst unüberbrückbar scheinende Gegensatz von Selbstinteresse und Unterwerfung aufgelöst werden. Im Detail verschieden, aber mit ähnlicher

28 S. auch die Berücksichtigung des Foole bei Gauthier (1986) 160 ff; (1990) 17 ff, 65; (1993) 28 sowie den Aufsatz von T. Schmidt in diesem Band.

29 Hampton (1986) Kap. 8. 
Tendenz ersetzt Kavka den Hobbesschen Naturzustand durch eine der Rawlsschen ähnlichen Ursituation und die absolute Herrschaft durch einen minimalistischen Wohlfahrtsstaat. ${ }^{30}$

Diese Rekonstruktionsversuche verdeutlichen zweierlei: Erstens, das Akzeptieren einer uneingeschränkten Zwangsgewalt, wie von Hobbes vorgesehen, ist auf der Grundlage eines auch nur leicht entdramatisierten Naturzustands rational nicht mehr nachvollziehbar. Wer nicht akut und beharrlich der Todesdrohung ausgesetzt ist, hat keinen guten Grund, sich einer friedensstiftenden, aber willkürlichen Macht anzuvertrauen. Die bei Hobbes etwas versteckte Stimme des Foole transformiert die Hobbessche alles-oder-nichtsArgumentation in eine notwendig differenzierte individuelle Nutzenkalkulation, die mit einer Unterwerfung unvereinbar ist.

Zweitens, ein von Selbstinteresse ausgehendes hobbesianisches Argument muß in irgendeiner Weise einen Einstellungswandel umfassen. Minimal könnte dieser Wandel einfach darin bestehen, daß die Beteiligten sich der konflikthaften Situation und des kollektiv unzweifelhaft bestehenden rationalen Zwangs für eine Friedensordnung bewußt sind. Wirksamer wäre aber ein Wandel in den Motiven selbst. In beiden Fällen spricht allerdings nichts dafür, daß eine Zwangsordnung in Form eines isolierten Rechte-Regimes eingeführt werden kann, das mit der Eigenmotivation der Beteiligten im täglichen Handeln in keiner Weise verknüpft ist. Nichts spricht also dafür, daß das Hobbessche Argument in Form einer kollektiv-einmaligen Selbstfesselung erfolgreich sein könnte. Hobbesianische Begründungen müssen vielmehr sowohl individualistisch wie dem Ziel nach nicht rechteorientiert, sondern moralorientiert sein. Erfolgversprechendere Ansätze wie der von Gauthier oder von Stemmer sind beides, im Unterschied zu Hobbes' Staatstheorie sind es also genuine Ansätze innerhalb der Moralphilosophie.

\section{Gauthier}

Gauthiers Ansatz beruht auf zwei Vermutungen, einer zur Möglichkeit der rationalen Einigung und einer zur Stabilität des rationalen Befolgens. Gegenüber dem Naturzustand hegt Gauthier die Vermutung, daß selbst wenn man ihn (wie Hobbes) als einmaliges Gefangenendilemma betrachtet, das im Prinzip die Möglichkeit des rational vorteilhaften Kooperierens nicht ausschließt. Eine solche Möglichkeit müsste, so die Vermutung, mithilfe einer präzisierten Kooperationstheorie formulierbar sein. Und gegenüber dem nachfolgenden

30 Kavka (1986) Kap. 4 und 5; für eine auführlichere kritische Diskussion der Bücher von Hampton und Kavka s. Kraus (1993). 
Befolgen rational vorteilhafter Normen erwartet Gauthier, daß sie über eine dispositionale Änderung der Präferenzen auch als rational-stabil ausgewiesen werden kann. In Morals by Agreement (1986) sind diese beiden theoretischen Vermutungen in detaillierte Diskussionsvorschläge umgesetzt worden.

Gauthiers Theorie rationaler Einigungen besteht wiederum in zwei Teiltheorien. Jede Einigungssituation lässt sich nämlich unter zwei Aspekten zerlegen. Erstens unter der Frage, nach welcher Entscheidungslogik die Beteiligten in der Situation, die Voraussetzungen dieser Situation einmal gegeben, zu einem Ergebnis gelangen. Und zweitens unter der Frage, welche Voraussetzungen der Situation als solcher akzeptiert werden sollen. Offensichtlich sind beide Punkte für eine sich selbst moralisch voraussetzungslos verstehende Begründung der Moral bedeutsam: Nach welcher Logik können oder müssen sich die Egoisten auf ein für alle vorteilhaftes Ergebnis einigen bzw. sind auch höchst ungleiche Ausgangsbedingungen akzeptabel? Hobbes nahm einfach an, daß im Naturzustand jeder jedem gegenüber gleich bedrohlich ist (Lev. XIII, 1); aber warum sollte die reale Bedrohung nicht entsprechend variieren, da kaum alle Menschen gleich stark, gesund oder intelligent sind? Und ist dann - eine endemische Frage des realistischen Kontraktualismus - ein nichtegalitäres Moralverständnis nicht unvermeidlich?

Gauthier hat ebenso originelle wie in ihrem effektiven Ertrag anhaltend umstrittene Lösungen zu diesen Teilproblemen vorgeschlagen. Seiner Meinung nach wird eine rational zwingende Entscheidungslogik für die Egoisten anhand einer speziellen Variante der neueren Verhandlungstheorie geliefert. ${ }^{31}$ Sein Vorschlag einer Lösung mittels ,minimax-relativer Konzessionen“ besteht der Grundidee nach darin, eine Gefangenendilemma-Situation in ideale Verhandlungen zu überführen, in denen eine Kooperationslösung nach der Formel angestrebt werden soll: „Minimiere das Maximum deiner Konzessionen gegenüber deinen Verhandlungspartnern!“ Diese Einigungslogik soll einen doppelten Vorteil haben, nämlich nicht nur allen Beteiligten den Kooperationsgewinn sichern, der im nicht-kooperativen Ergebnis des Gefangenendilemmas verloren geht, sondern außerdem noch gegenüber individuell ungleichen Kooperationsanteilen sensibel sein, also ein Element der Fairness berücksichtigen.

Hobbes‘ pauschales Gleichheitsdogma ersetzt Gauthier durch die insgesamt erstaunlichste seiner auf verschiedenen Niveaus optimistisch-rationalen Argumente, nämlich die rein nutzenrationale Geltung des ,Lockeschen Provisos $^{6}{ }^{32}$ Mit ,Lockes Proviso' wird auf Lockes Bedingung angespielt, wonach die Aneignung von Naturgütern als Privatbesitz nur dann gerechtfertigt ist,

31 Gauthier (1986) Kap. 5.

32 Ebd. Kap. 7. 
wenn noch genügend und gleich wertvolle Naturgüter für die zum gleichen Zeitpunkt Besitzlosen übrig sind. Mit dieser Einschränkung soll die Idee der gleichen Rechte aller Menschen auf Naturgüter gefaßt werden. Wird eine solche Einschränkung anders als bei Locke (oder R. Nozick) nicht selbst bereits moralisch verstanden, muß der Nachweis erbracht werden, daß auch rationale Egoisten ein Lockesches Proviso akzeptieren würden. Gauthiers Proviso fordert, daß die Ausgangsposition für Verhandlungen nicht auf Ergebnissen beruht, die durch Zwang entstanden sind. Anders gesagt fordert er ein gewisses $\mathrm{Ma}$ an Freiheitsrechten in der Verhandlungssituation. Rational-optimistisch ist Gauthier darin, daß er behauptet, der Zwang sei proportional nutzenhinderlich, oder umgekehrt, Freiheit nutzenförderlich. Unter dieser Bedingung würden vielleicht nicht inhaltlich völlig gleiche, aber immerhin annähernd gleiche und insbesondere zwangsfrei akzeptierte Normen ermittelt.

Der Foole bestreitet, daß selbst rational ermittelte Normen befolgt werden sollten, gegeben seine individuell-egoistische Sicht. Gauthier antwortet hierauf mit einer Befolgenstheorie (seiner zweiten Theorie), in der die bereits erwähnte Idee des Dispositionenwandels präzisiert wird. ${ }^{33}$ Gauthiers Idee ist im Grunde naheliegend: Der Kooperationsgewinn für alle hängt in Gefangenendilemma-Situationen davon $a b$, daß sie gegenseitig glaubwürdig sind, glaubwürdig können sie aber nur sein, wenn sie gegenseitig als glaubwürdig gelten oder wenn sie verläßlich glaubwürdig sind. Eine solche Glaubwürdigkeit würde entstehen, wäre gesichert, daß die Akteure über eine feste Disposition des Normenbefolgens verfügen, daß sie, wie Gauthier sagt, dispositionell (in ihren Handlungseigenschaften) ,beschränkte und nicht , uneingeschränkte‘ Nutzenmaximierer sind. Was der Foole übersehen hat, ist neben der auch auf ihn zurückfallenden Kooperationseinbuße die Möglichkeit, in Übereinstimmung mit geeigneten Partnern Kooperationsdispositionen anzunehmen und beizubehalten, um so den Kooperationsgewinn durch Verläßlichkeit des erwartbaren Verhaltens sowohl gemeinsam wie individuell zu nutzen.

In Ausarbeitung dieser Grundidee versucht Gauthier ein exakteres wahrscheinlichkeitstheoretisches Argument dafür zu geben, daß es unter annähernd realistischen Bedingungen individell-nützlicher ist, eine kooperative als eine nicht-kooperative Disposition anzunehmen. Die Pointe seines Nachweises besteht darin, daß von einem bestimmten Anteil kooperativ Disponierter in der Gemeinschaft an der Vorteil dadurch, selbst als kooperativ Disponierter erkannt zu werden, größer ist als der Nachteil, von einem Nichtkooperierer ausgebeutet zu werden. ${ }^{34}$ Wo das genaue Maß dieses Anteils liegt, ist nicht sicher, aber immerhin scheinen sogar in einer gleichmäßig in Kooperierer und Nichtkooperierer aufgeteilten Gemeinschaft die Kooperierer noch Koopera-

33 Ebd. Kap. 6

34 Ebd. 176 f. 
tionsvorteile zu haben, wenn sie häufiger mit Kooperierern als Nichtkooperierern zusammenzuarbeiten. Da das Kooperieren dispositionell festgelegt ist, kann die Auswahl zwischen diesen beiden Kollektiven nicht mehr bewußt gesteuert werden, sondern muß sich als vorteilhafter sozialer Zustand ergeben. 35

Positiv läßt sich von Gauthiers Ansatz sagen, daß in ihm alle Schwierigkeiten einer rein rationalen Vertragstheorie erkannt und mit subtilen Argumenten beantwortet werden. Negativ gilt wohl, daß es sich eher um ein offenes Forschungsprojekt als um ein auch nur teilweise wirklich überzeugendes oder gar zwingendes Unternehmen handelt. ${ }^{36}$ Die minimax-relativen Konzessionen als Lösung des Verhandlungsspiels sind als formales Angebot nicht eindeutig zwingend. Daß die Zwangsfreiheit als Voraussetzung des Verhandelns rein nutzenrational belegt werden könnte, wirkt bis heute wenig überzeugend. Die Befolgenstheorie ist hoch-idealisiert. Das Gesamtprojekt ist außerdem auf den Erfolg jeder dieser Teiltheorien angewiesen. Versagt auch nur eine, kann von der Begründung einer auch nur halbwegs egalitären Moral aus dem bloßen Selbstinteresse heraus nicht gesprochen werden.

\section{Stemmer}

P. Stemmers aktueller Versuch einer Vertragsbegründung der Moral in Handeln zugunsten anderer (2000) teilt mit Gauthiers Ansatz dessen hobbesianische Grundhaltung, unterscheidet sich von ihm jedoch durch eine andere Schwerpunktbildung und, damit verbunden, eine unterschiedliche Methodik. Gauthier kollektiviert gleichsam den Foole von vornherein, indem er sich der Möglichkeit einer rational-selbstinteressierten Moral über das Marktversagen und die Mängel des Marktmodells für die Gesellschaft insgesamt nähert. ${ }^{37}$ Strategische Handlungsanalysen mithilfe der Spieltheorie setzen diese Perspektive natürlicherweise fort. Stemmer legt seine Argumentation hingegen als einen fortgesetzten Dialog mit einem paradigmatisch gewählten rationalen Egoisten an und diese singuläre Perspektive bleibt soweit dominant, daß auf spieltheoretische wie überhaupt kollektiv-soziale Analysen weitgehend verzichtet wird.

35 Das Studium einer entweder selbststabilisierenden oder selbstdestruktiven Dynamik kooperierender Akteure ist für Gauthiers Ansatz deshalb wichtig. Ein Klassiker zu dieser Frage ist Axelrod (1984). Allerdings ist meines Erachtens ein Urteil über die soziale Aussagekraft dieser Evolutionstheorie im Augenblick noch schwer möglich. Vgl. hierzu auch die Arbeiten in Analyse \& Kritik 1/2000.

36 Kritische Diskussionen von Gauthiers Teiltheorien sind insbesondere enthalten in Vallentyne (ed.) (1991); Gauthier/Sudgen (eds.) (1993); Kraus (1993); Stemmer (2000) 233-246.

37 Gauthier (1986) Kap. 4. 
Stemmer hat ein anderes Ziel. Während die meisten stark-deskriptiven Kontraktualisten, in Verbindung mit ihrem Schwerpunkt in Sozialanalysen, an der genaueren Struktur moralischer Gebote oder Rechte meist wenig interessiert sind, oder Moral sogar häufig einfach mit ,sozialen Normen gleichsetzen, versucht Stemmer vielmehr vor allem eine genauere Analyse des moralischen Sollens im Rahmen einer kontraktualistischen Argumentation vorzulegen. Dazu dient neben einer formalen Analyse dieses Sollens mithilfe selbstauferlegter Sanktionen vor allem auch ein inhaltlicher Überblick zu den konkreten Merkmalen einer kontraktualistischen Moral.

Der Titel des Buchs ist inklusiv gemeint. Ähnlich wie Hume die ,natürlichen' von den ,künstlichen' Tugenden unterschied, die ersteren auf direkt wirkenden Affekten wie dem Wohlwollen beruhen sah, die letzteren auf dem indirekt wirksamen Selbstinteresse (T, III.ii-iii), unterscheidet Stemmer einen altruistischen und einen egoistischen Bereich, wobei er, vielleicht etwas irritierend, nur den letzteren denjenigen der Moral nennt. Das Handeln ,zugunsten anderer' umgreift also moralisches und altruistisches Handeln und eine Einsicht von Stemmers Ansatz soll es sein, zu erkennen, wie wenig die Moral allein eigentlich bewirken kann bzw. wie sehr sie durch den Altruismus ergänzt werden muß. ${ }^{38}$

Dieser terminologischen und inhaltlichen Unterscheidung der zwei Bereiche zugrunde liegt eine für die Argumentation des Buchs zentrale Unterscheidung zwischen rational zwingenden und rational möglichen praktischen Gründen. ${ }^{39}$ Stemmer beginnt seine Analyse als Gespräch mit einem moralischen Skeptiker, der als Skeptiker, wie Stemmer meint, nur egoistisch-vorteilhafte Gründe gelten lassen kann. Nur die den Egoisten befriedigenden Gründe sind folglich rationale Gründe, nur die von ihm als zwingend anzuerkennenden Gründe sind ,rational zwingende' Gründe. Rational zwingende Gründe für den Altruismus kann es dementsprechend nicht geben, und Stemmer versucht insbesondere am Mitleid zu zeigen, daß es zwar begrüßenswert bzw. rational möglich, aber nicht zwingend sein kann. ${ }^{40}$

Stemmers Hoffnung auf den Altruismus ist verständlich. Denn die rational-zwingende, also vom Egoisten zu akzeptierende Moral ist gegenüber unserem Alltagsverständnis inhaltlich schockierend karg. Es liegt generell nahe, daß eine auf wechselseitigem Vorteil beruhende Moral den Schwachen einen geringeren Schutz gewährt als den Starken - also gerade dem zuwiderläuft, was wir in der christlichen Alltagsmoral für angemessen halten. Auch Gau-

38 Stemmer (2000) 295, 346, 375.

39 Ebd. 15, 29, 33 f., 63 f., 67, 147, 303-7.

40 Ebd. $\$ 10$. 
thier hat das eingestanden, durch idealisierende Korrekturen wie die fairen Ausgangsbedingungen des Verhandelns und einen, Archimedischen Punkt ${ }^{`}$ der Unparteilichkeit jedoch zugunsten einer weitgehenden Gleichheit zu korrigieren versucht. ${ }^{41}$ Stemmer kritisiert Gauthiers nutzenrationales Plädoyer für ein Lockesches Proviso ${ }^{42}$ und reduziert dementsprechend die rational zwingende Moral auf einen Kernbereich von besonderen Lebewesen: Menschen, die relativ gesehen gleich stark und kooperationsfähig sind. Die rational zwingende Moral ist seiner Meinung nach weder universalistisch noch egalitär. Nicht nur die Tiere, auch permanent handlungs- und kooperationsunfähige Menschen fallen aus ihr heraus, und diejenigen, die von ihr erfaßt werden, finden sich eingestuft entsprechend dem Ausmaß ihrer aktuellen Fähigkeiten.

Angesichts des Pathos und der sozialen Bedeutung, die in unserer öffentlichen westlichen Kultur mit moralischen Begriffen wie „Menschenrechten“ oder „Menschenwürde“ verbunden sind, wird angesichts der philosophischen Verteidigung einer so radikalen Auffassung von Moral die Frage spannend, wie ihr Vertreter seine Leser und Kollegen von der Unausweichlichkeit seiner Position überzeugen will. Dies betrifft auf der einen Seite das argumentative Spiel im engeren, technischen Sinn, in dem argumentativ überzeugend gezeigt werden muß, daß Fragen und Antworten genau die Analyse auszeichnen, die vorgeschlagen wird. ${ }^{43}$ Auf der anderen Seite muß sich unser alltägliches Selbstverständnis in einer philosophischen Analyse zur Moral, im Unterschied vielleicht zur Analyse von genuin philosophischen Gegenständen wie Substanz oder Supervenienz, irgendwie wiederfinden. Ob Stemmers Analyse beide Anforderungen zu erfüllen vermag, ist das Thema der meisten Artikel in diesem Band und kann in seinen Details hier nicht vorweggenommen werden.

Zum Teil hat sich Stemmer, indem er den Bereich des Altruismus neben dem der rationalen Moral zugestanden hat, von den kontraintuitiven Folgen seiner Theorie sicher entlastet. Wie erfolgreich dieser Zug zu sehen ist, hängt allerdings von den Erwartungen ab, die mit der Moralbegründung bzw. dem Argumentieren gegenüber dem Egoisten verbunden sind. Sollen wir die

41 Gauthier (1986) 268 und Kap. 8.

42 Stemmer (2000) 234-248.

$43 \mathrm{Da}$ wir zur Formulierung dieser Erfolgsbedingungen auf einen weiteren, nämlich argumentativen Begriff des rationalen Zwangs zurückgreifen dürfen und müssen, im Unterschied zu Stemmers ,rationalen Zwang' nach Maßgabe des Egoisten, ist ein subtiles Anzeichen dafür, daß der Kontraktualismus die vorhin als wünschenswert herausgestellte Synthese von Moral und Politik, oder von Modell und öffentlicher Verteidigung, schwer erbringen wird können. Warum messen wir die Stimmigkeit der Argumentation nicht selbst wiederum an egoistischen Maßstäben? 
altruistischen Motive einfach akzeptieren, auch wenn sie unbegründet sind, oder sind sie hinreichend begründet, wenn sie einfach ,rational möglich ${ }^{6}$ sind? Benötigen sie keine Begründung, warum dann das moralische Handeln? Warum die Suche nach einem rationalen Zwang, von dem dann ein Teil der Motive ausgenommen wird? Es scheint, daß von einem Standpunkt aus die einen Motive abgewertet werden müssen, wenn die anderen derart aufgewertet werden. Nicht rational zwingend zu sein, sollte ein Makel für den Altruismus werden, bzw. wenn nicht, sollte der Egoismus nicht als rational vorteilhaft erscheinen können. Ob die von Stemmer erwünschte Synthese von Moral und Altruismus möglich ist, wäre also erst zu zeigen.

Zweitens, und wichtiger, bietet Stemmers Ansatz eine zusätzliche Angriffsfläche im Vergleich etwa zu Gauthier, insofern er dem Ziel nach nicht einfach ,rationale Normen' oder ,soziale Normen', sondern moralische Normen, ein moralisches Sollen, rechtfertigen will. Während die meisten Humeaner die Frage, was moralische Normen von anderen unterscheidet, als irrelevante Frage ansehen, will Stemmer vorrangig unser Verständnis von Moral analysieren, genauer es als rational zwingend analysieren. Dem entspricht das Gleichsetzen des Begriffs der Moral mit dem ,rational zwingenden“ Handeln gegenüber anderen. Nun ist unser alltäglicher Begriff des moralischen Sollens sicher nicht gleichbedeutend mit einem Sollen, das dem Egoisten gegenüber rational zwingend ist - im Gegenteil dürften wir meist der Meinung sein, daß gerade das Gegenteil der Fall ist. Die Moral soll nach üblichem Verständnis den Egoismus korrigieren, nicht ihn bedienen. Wie kann Stemmer diesen Konflikt mit unserem alltäglichen Selbstverständnis beantworten?

Stemmers Rekonstruktion des moralischen Müssens ist sowohl als eine metaethische Analyse, wie als eine Begründung dafür gemeint, warum es für Egoisten nützlich ist, bestimmte moralische Rechte und Pflichten anzuerkennen. ${ }^{44}$ In Anschluß an frühere Vorschläge von H. Kelsen und E. Tugendhat identifiziert Stemmer das moralische Müssen mit einem in hypothetischer Übereinstimmung mit anderen festgelegten, also ,künstlich geschaffenen Sanktionsrecht. Die moralische Pflicht wird also mit der Sanktionierbarkeit eines Nichteinhaltens der Pflicht in moralisch relevanten Situationen identifiziert. Die moralische Qualität dieses Müssens, im Unterschied etwa zum erpresserischen Zwang seitens eines Kriminellen, beruht auf der hypothetisch gedachten autonomen Zustimmung zu einer Norm für eine offene Zahl von Handlungssituationen. ${ }^{45}$

44 Stemmer $(2000) \mathbb{} \$$.

45 Ebd. 108-110; in diesem Band 42, 59-68. 
Die angesprochene Übereinstimmung kann nicht anders als hypothetisch verstanden werden. ${ }^{46}$ Stemmer geht sogar soweit, die Rede vom Vertrag für bedeutungslos $\mathrm{zu}$ halten. ${ }^{47}$ Dennoch muß die hypothetisch angesonnene Übereinkunft eine sein, der reale Interessen zugrundeliegen. Im Fall elementarster Gebote wie Nichttöten oder Nichtstehlen mag es trivial erscheinen, die entsprechenden Interessen zu unterstellen, aber bereits beim Lügenverbot ist unklar, auf welches nicht bereits moralisch zu beschreibende Interesse man sich beziehen soll. Und sowieso würde eine stärker differenzierte Moral zunehmend riskante empirische Behauptungen über reale Interessen aufstellen müssen, also in einen stark-deskriptiven Kontraktualismus übergehen. Der deskriptive Gehalt der hypothetischen Unterstellung von Sanktionsinteressen ist deshalb nicht unbeachtlich.

Für Stemmers speziellen Vorschlag der Identifikation des moralischen Müssens mit potentiellen Sanktionen sind aber drei andere Fragen einschlägig. Erstens, ist die Identifikation des Moralischen mit Sanktionen nicht widersprüchlich, da wir üblicherweise moralisch gerade nicht der Sanktionsdrohung wegen handeln? Letzteres würde das erstere ausschließen.

Zweitens, worin bestehen eigentlich genauer die Sanktionen, die man beim Nichteinhalten der Pflichten zu beachten hat? Wie die Übereinkunft hypothetisch ist, so offensichtlich auch die Sanktionsdrohung. Dennoch muß die hypothetische Überlegung, auch was die Sanktionen betrifft, in den Gepflogenheiten der realen Gesellschaft irgendwie verankert werden, ähnlich wie hinsichtlich der Interessen. Insofern müssten die bereits real wirksamen Sanktionen des unmoralischen Handelns als Vehikel für die rational gebotenen Sanktionen verstanden werden. Ist das aber möglich?

Damit in Verbindung steht, drittens, eine nötige Antwort auf die Position des Foole. Der Foole wird nämlich gegenüber diesem Vorschlag einwenden, daß er mit den realen Sanktionen so umzugehen gedenkt, wie er sie eben real zu befürchten hat. Da die realen Sanktionsdrohungen vermutlich nicht ausreichend sind, weil die Alltagsmoral stark eigenmotiviert angelegt ist und viele Verstöße unentdeckt bleiben, dürfte sich der Foole häufig dem ,rationalen Zwang' der Sanktionen entziehen können. Da der Foole eine repräsentative Figur ist, wird fraglich, inwiefern die rationale Moral Bestand haben kann.

Die erste Frage könnte Stemmer wie alle naturalistischen Kontraktualisten mit dem Hinweis beantworten, daß es ihm eben darum gehe, die traditionelle Moral rational zu rekonstruieren, und das bringe eine veränderte Motivation

46 S. ähnlich Kavka (1986) 22; Stemmer in diesem Band $61 \mathrm{f}$.

47 Stemmer (2002) $9 \mathrm{f}$. 
mit sich. Sich demgegenüber nur auf den Begriff der traditionellen Moral zu beziehen, bedeute, die Begründung zu verweigern. In diesem Punkt führen Frage und Antwort zu einem Patt-Zustand. Auch Stemmers Antwort wäre nicht völlig überzeugend. Stemmer geht es ja darum, das moralische Müssen zu rekonstruieren, so daß sich die traditionelle Moral nicht einfach unter Verweis auf die Art des Begründens beliebig ersetzen oder transformieren läßt.

Was die Frage des Anbindens der hypothetisch gebotenen Sanktionen an eine bereits bestehende ,Sanktionskultur' betrifft, ist Stemmer eher zurückhaltender als andere Kontraktualisten. Tugendhat beispielsweise hat in seiner Skizze kontraktualistischer Argumente neben den äußeren Sanktionen auch ,innere' Sanktionen anhand der moralischen Gefühle, vor allem Empörung und Scham, angeführt. Stemmer hält ein solches Rückführen des moralischen Müssens auf eine ,moralische Identität ${ }^{\star}$ und die mit ihr verbundenen Gefühle zwar für möglich, im Sinn seiner Unterscheidung jedoch nicht für rational zwingend. ${ }^{48}$ Psychische Dispositionen sind nicht ausreichend kontrollierbar, um zwingend sein zu können. Anders verhält es sich seiner Meinung nach mit Weisen des „sozialen Ausgrenzens“ oder der „sozialen Quarantäne“.49 Ersichtlich führt dieser Vorschlag weitere Fragen mit sich. Meines Erachtens betrifft das vor allem die Differenz zu Tugendhats Vorschlag. In der realen Gesellschaft müssen die Akte des Ausgrenzens ja ihrerseits motiviert sein, und die Motive werden kaum diejenigen von rationalen Egoisten sein, die ohne konkrete Gefühle handeln. Wie die Kluft zwischen der hypothetischen Überlegung und der realen Sanktionskultur überbrückt werden soll, bleibt damit offen.

Die dritte Frage, oder das Befolgensproblem, ist für Stemmer angesichts der eben geschilderten Zurückhaltung gegenüber moralischen Identitäten und negativen Affekten natürlich schwer befriedigend zu beantworten. Wenn ich es recht verstehe, ist Stemmer der Meinung, daß die praktischen Probleme eines ideal vorstellbaren ,Dispositionentauschs' ${ }^{6}$ unter Egoisten so erheblich sind, daß ein solcher Tausch nicht realisierbar wäre. ${ }^{50}$ Auf der anderen Seite schließt er das Etablieren „sekundärer Sanktionen“, die sich nicht auf Einzelhandlungen, sondern auf den moralischen Charakter einer Person richten, nicht aus, sondern befürwortet sie geradezu. ${ }^{51}$ Indem die ihrem Charakter nach erkennbar zum unmoralischen Handeln bereiten Personen sozial sanktioniert werden, soll die Lücke des „Handeln im Verborgenen“ zwar nicht prinzipiell, aber doch einigermaßen wirksam geschlossen werden.

48 Stemmer $(2000) \$ 5$.

49 Ebd. 152-161.

50 Ebd. $\$ 6$.

51 Ebd. 187. 
Diese Überlegungen beleuchten insgesamt die Dringlichkeit des Problems, wie man sich eine in hypothetischer Konstruktion durchdachte ,rationale Moral' in die reale Gesellschaft übersetzt denken soll. Der Kontraktualismus benötigt eine explizite ,Realisierungstheorie', in der einerseits studiert würde, wie sich der rationale Zwang unter hypothetischen Egoisten zum entsprechenden Zwang unter den Mitgliedern einer gegenwärtigen realen Gesellschaft verhält, und in der andererseits gezeigt werden müsste, daß die reale Gesellschaft den ihr angesonnenen rationalen Zwang zu realisieren vermag. Gauthiers und Stemmers Bemerkungen zu Dispositionen gehen in die Richtung einer solchen Theorie, bleiben aber offensichtlich noch lückenhaft. Wie bei Gauthiers Vermutung, die ,unbegrenzten Maximierer' könnten durch ,restringierte Maximierer ${ }^{6}$ ersetzt werden, bleibt auch in Stemmers zuletzt angesprochenem Vorschlag des Sanktionierens nicht moralisch motivierter Personen die offene Frage, wie es rationalen Egoisten gelingen soll, sich in solche Personen zu transformieren. Einander wegen dieses, Charakterdefizits' zu sanktionieren erscheint nicht vereinbar damit, ein rationaler Egoist zu sein.

\section{Vernunft, Moral und Politik}

Am Ende dieses Überblicks bleibt der Eindruck, daß beide Traditionen, die des Hobbesschen und des Kantischen Kontraktualismus, Schwierigkeiten haben, die Bürde des jeweils von ihnen gewählten Vernunftbegriffs vollständig zu tragen. Der vermeintlich starke kantianische Vernunftbegriff kann wohl nur durch eine erst auszuarbeitende Theorie der praktischen Gründe, entsprechend der Vorschläge von Scanlon und anderen, gegen die klassischen Einwände einer bloß rhetorischen Sicherung der traditonellen Moral verteidigt werden. Der kantianische Kontraktualismus scheint also eine weitere normative Theorie der Gründe zu benötigen. Der vermeintlich schwache instrumentelle Hobbessche Vernunftbegriff führt die mit ihm verbundenen kontraktualistischen Theorien hingegen eher dazu, die Realisierungsbedingungen des Diskurses unter rationalen Egoisten detaillierter formulieren zu müssen.

Sollte diese Beobachtung zutreffen, erwiese sich Hume als derjenige Vertreter der naturalistischen Vertragstheorie, der eine konsequente, und vielleicht einzig mögliche Lösung des Realisierungsproblems im Rahmen dieser Tradition eröffnet hat. Vielleicht sind Humes Konventionalismus bzw. die ihn heute erneuernden Studien in Anschluß an R. Axelrod die einzig relevanten Versionen der benötigten Realisierungstheorie für eine kontraktualistische Ethik. Wie bereits bei Hume verschärft sich dadurch natürlich die Frage, wie sich Rechtfertigen und Erklären, ideale Moral und reales Handeln zueinander verhalten. Wie kann der Rationalitätsanspruch dieser Theorie bewahrt bleiben, wenn sie in so hohem Maß auf die Wirksamkeit in realen Gemeinschaften angewiesen ist? 
Ich muß gestehen, daß ich alternativ einer ,kantianischen Realisierungstheorie' die größeren Chancen einräume. Anders als die instrumentelle Vernunft muß eine sich formal aus Argumenten und Gründen ergebende Vernunft keine prinzipielle Kluft zwischen Egoismus und Moral, Motivation und Zwang überbrücken, und ist ihrer Grundbegriffe wegen von vornherein darauf angelegt, öffentlich zu sein. Sicher muß auch ein kantianisches Rechtssystem berücksichtigen, daß moralisch geforderte Verhältnisse erzwungen werden müssen. Aber es bedeutet in diesem System keinen internen Konflikt, dies zu tun. Die Kantisch verstandenen Rechte sind nicht nur Fesseln des Egoismus, sondern zugleich eine moralische Anerkennung der anderen als Rechteinhaber, und in dieser Zugabe steckt eine Quelle von Friedensmöglichkeit, die der Hobbes-Humeschen Tradition nicht verfügbar ist.

Hume hat vermutlich darin recht, daß die reale Gesellschaft der letzte und definitive Prüfstein für die gelingende Moralbegründung, oder für eine vernünftige Moral ist, er war nur einseitig der Meinung, dieser Zustand würde sich anhand der Gefühle und Sitten einspielen. In einer aktuellen westlichen Gesellschaft ist eher zu sehen, daß sich die selbst moralisch verstehende Gesellschaft zumindest nicht unabhängig von moralischen Argumenten herstellt, ohne die Macht der Argumente dabei überzubewerten. Wenn die tatsächliche Entwicklung eine Alternative auszeichnen wird, so sollte es dennoch eine sein, die von der Vernunft vielleicht nicht erzwungen, aber von ihr durch Verallgemeinerung auch nicht zerstört wird.

\section{Literatur}

Hobbes, T. (Lev.), Leviathan, 1651.

Hume, D. (T), A Treatise of Human Nature, 1739-40.

Hume, D. (C), Of the Original Contract, 1748.

Kant, I. (Idee), Idee zu einer allgemeinen Geschichte in weltbürgerlicher Absicht, 1784.

Kant, I. (GMS), Grundlegung zur Metaphysik der Sitten, 1785.

Kant, I. (KU), Kritik der Urteilskraft, 1790.

Kant, I. (Gemeinspruch), Über den Gemeinspruch: Das mag in der Theorie richtig sein, taugt aber nicht für die Praxis, 1793.

Kant, I. (Frieden), Zum ewigen Frieden, 1795.

Kant, I. (MS-RL), Metaphysik der Sitten, Rechtslehre, 1797.

Kant, I. (Anth), Anthropologie in pragmatischer Hinsicht, 1798

Rawls, J. (TG), Eine Theorie der Gerechtigkeit, Frankfurt 1975; orig. A Theory of Justice, Cambridge, Mass. 1971.

Rawls, J. (JF), Justice As Fairness. A Restatement, Cambridge, Mass. 2001. 
Axelrod, R. (1984), The Evolution of Cooperation, New York/London; dt. Die Evolution der Kooperation, München ${ }^{3} 1995$.

Binmore, K. (1994), Game Theory and the Social Contract, Vol. 1: Playing Fair, Cambridge, Mass.

Boucher, D./Kelly, P. (1994), The Social Contract and its Critics. An Overview, in: Boucher/Kelly (eds.) (1994) 1-34.

Boucher, D./Kelly, P. (eds.) (1994), The Social Contract from Hobbes to Rawls, London/New York.

Buchanan, J. M. (1975), The Limits of Liberty. Between Anarchy and Leviathan, Chicago.

Dworkin, R. (1977), Justice and Rights, in: R. D., Taking Rights Seriously, New York, 150-183.

Euchner, W. (1979), Naturrecht und Politik bei John Locke, Frankfurt.

Gauthier, D. (1986), Morals By Agreement, Oxford.

Gauthier, D. (1988), Hobbes' Social Contract, in: G. A. Rogers/A. Ryan (eds.), Perspectives on Thomas Hobbes, Oxford, 125-152.

Gauthier, D. (1990), Moral Dealing. Contract, Ethics, and Reason, Ithaca.

Gauthier, D. (1993), Between Hobbes and Rawls, in: Gauthier/Sudgen (eds.), 24-39.

Gauthier, D. (1997), Political Contractarianism, in: P. Koller/K. Puhl (eds.), Current Issues in Political Philosophy: Justice in Society and World Order, Wien, 21-34.

Gauthier, D./Sugden, R. (eds.) (1993), Rationality, Justice and the Social Contract. Themes from Morals by Agreement, New York.

Goldsmith, M. M. (1966), Hobbes' Science of Politics, New York.

Gough, J.W. (1936), The Social Contract, Oxford (2. Aufl. 1957).

Hampton, J. (1986), Hobbes and the Social Contract Tradition, Cambridge.

Harman, G. (1988), Rationality in Agreement, in: Social Philosophy and Policy 5, 1-16.

Harman, G. (1996), Moral Relativism, in: G. Harman/J. Thomson, Moral Relativism and Moral Objectivity, Oxford, 1-64.

Hayek, F. (1982), Law, Legislation and Political Economy, 3 Vols., London.

Kavka, G. (1986), Hobbesian Moral and Political Theory, Princeton.

Kraus, J. (1993), The Limits of Hobbesian Contractarianism, Cambridge.

Lewis, D. (1969), Convention. A Philosophical Study, Cambridge, Mass.

Maus, I. (1998), Der Urzustand, in: O. Höffe (Hg.), John Rawls. Eine Theorie der Gerechtigkeit, 71-95.

Mitsis, P. (1988), Epicurus' Ethical Theory, Ithaca/London.

Nagel, T. (1987), The View from Nowhere, New York.

Nozick, R. (1974), Anarchy, State, and Utopia, Oxford.

Popkin, R. (1979), The History of Scepticism from Erasmus to Spinoza, Berkeley. 
Popkin, R. (1982), Hobbes and Scepticism, in: R. P. (ed.), History and Philosophy in the Making, Washington.

Ridge, M. (2001), Saving Scanlon: Contractualism and Agent Relativity, in: The Journal of Political Philosophy 9, 472-481.

Scanlon, T. M. (1998), What We Owe to Each Other, Cambridge, Mass.

Schmidt, T. (2000), Die Idee des Sozialvertrags, Paderborn.

Schneewind, J. (1998), The Invention of Autonomy. A History of Modern Moral Philosophy, Cambridge.

Stemmer, P. (2000), Handeln zugunsten anderer, Berlin/New York.

Stemmer, P. (2002), Moralischer Kontraktualismus, in: Zeitschrift für philosophische Forschung 56, 1-21.

Tuck, R. (1979), Natural Rights Theories, Cambridge.

Tugendhat, E. (1993), Vorlesungen über Ethik, Frankfurt.

Vallentyne, P. (ed.) (1991), Contractarianism and Rational Choice. Essays on David Gauthier's Morals by Agreement, New York.

Watkins, J. W. N. (1965), Hobbes's System of Ideas, London.

Wolff, R. P. (1977), Understanding Rawls: A Reconstruction and Critique of A Theory of Justice, Princeton. 